\title{
A Review on Cuprate Based Superconducting Materials Including Characteristics and Applications
}

\author{
Md. Atikur Rahman ${ }^{1, *}$, Md. Zahidur Rahaman², Md. Nurush Samsuddoha ${ }^{3}$ \\ Department of Physics, Pabna University of Science and Technology, Pabna-6600, Bangladesh \\ Email address: \\ atik0707phy@gmail.com (Md. Atikur Rahman) \\ To cite this article: \\ Md. Atikur Rahman, Md. Zahidur Rahaman, Md. Nurush Samsuddoha. A Review on Cuprate Based Superconducting Materials Including \\ Characteristics and Applications. American Journal of Physics and Applications. Vol. 3, No. 2, 2015, pp. 39-56. \\ doi: 10.11648/j.ajpa.20150302.15
}

\begin{abstract}
A general review on cuprate based superconducting materials has been made. The special features of cuprate based high-temperature superconductors have been explained from the material side and the physical properties side. The present statuses on applications of cuprate based high-temperature superconductors have been included. In this paper, all characteristics of cuprate based superconductors depending upon the recent discoveries and applications of those compounds have been included and explained so that a researcher can get a good idea about this field easily. The article gives a summary of the prevailing arguments of researchers to relate the material to cuprates and also the comparative features of many families of superconductors. The existing challenges, such as flux pinning, inter-grain Josephson losses due to population of voids and attempts towards the solution of these problems have been made by researcher have also been discussed. Since the high temperature superconductivity was discovered in $\mathrm{La}_{2-\mathrm{x}} \mathrm{Ba}_{\mathrm{x}} \mathrm{CuO}_{4}$ in 1986 , many research works have been done on this topic, but in this review paper we emphasis cuprate based material and we noted all properties in dividing many section and also made a comparison between different materials.
\end{abstract}

Keywords: Superconductors, Cuprate-Based Superconductors, Applications

\section{Introduction}

Superconductivity is a phenomenon of exactly zero electrical resistance and expulsion of magnetic fields occurring in certain materials when cooled below a characteristic critical temperature. It is a macroscopic quantum phenomenon. Its special physical properties have attracted the attention of many physicists and electric engineers, since it was discovered by Onnes in 1911. Superconductivity with its flow of electric current without friction amounts to the realization of the old human dream of a perpetuum mobile. The ratio of resistance between the normal-conducting and the superconducting ("SC") state has been tested to exceed 1014, i.e., it is at least as large as between a usual insulator and silver as the best normal-conducting material ${ }^{1}$. As the progress of cooling technique gave access to lower and lower temperatures, superconductivity established as common low-temperature instability of most, possibly all metallic systems (see Fig. 1). However, even though many types of superconductors were discovered for many years, the critical temperature was below $20 \mathrm{~K}$, thus, the uses of superconductor's required cooling using liquid helium. After $\mathrm{Nb}_{3} \mathrm{Ge}\left(T_{c}=23.2 \mathrm{~K}\right)$ was discovered in 1973 , the critical temperature did not increase by even $1 \mathrm{~K}$ for more than 10 years. Through the 1970s and 1980s, several types of experiments and theoretical discussions were carried out to discover "high-temperature superconductivity". It is important to remember what happened in this prehistoric era. Twenty seven years have passed since the first sample of high temperature superconductivity was discovered in $\mathrm{La}_{2-}$ ${ }_{x} \mathrm{Ba}_{\mathrm{x}} \mathrm{CuO}_{4}$ in 1986. Since then, many scientists rushed to these fields, as a result more than two hundred new materials were discovered, including many modifications of earlier materials and many types of high-temperature superconductors have been found in cuprate materials. Due to enormous research effort of a large number of physicists, chemists, and material scientists, the generic physical properties of those cuprate materials have been studied with high precision by applying various experimental methods. From these research efforts, it has emerged that these compounds possess a number of unusual normal state and superconducting properties due to a complicated interplay of electronic spin and lattice degrees of freedom. At present, there are more than 150 superconducting compounds with $T_{c}$ higher than the record of $23 \mathrm{~K}$ for 
conventional superconductors but only cuprates can be called the true high-temperature superconductors since only they have $T_{c}$ above the liquid-nitrogen boiling point and many of them even have $T_{c}>100 \mathrm{~K}$. However for practical application of superconductors we need superconductors with higher critical temperature and cuprate is best for this. A superconductor can be distinguished by following two properties: (i) Vanishing of electric resistivity below a critical temperature $\left(T_{c}\right)$. (ii) Expulsion of external magnetic field below a critical field $\left(H_{c}\right)$. In cuprate superconductor electric conduction arises from the pairing of charge carriers into copper pairs ${ }^{2}$. Superconductivity is believed to be a universal phenomenon, and is a collective state existing in electron population of a material. From superconducting cosmic strings created as topological defects in the early universe ${ }^{3}$ or proton superconducting cores in neutron $\operatorname{stars}^{4}$ to color superconductivity in quark matter. ${ }^{5}$

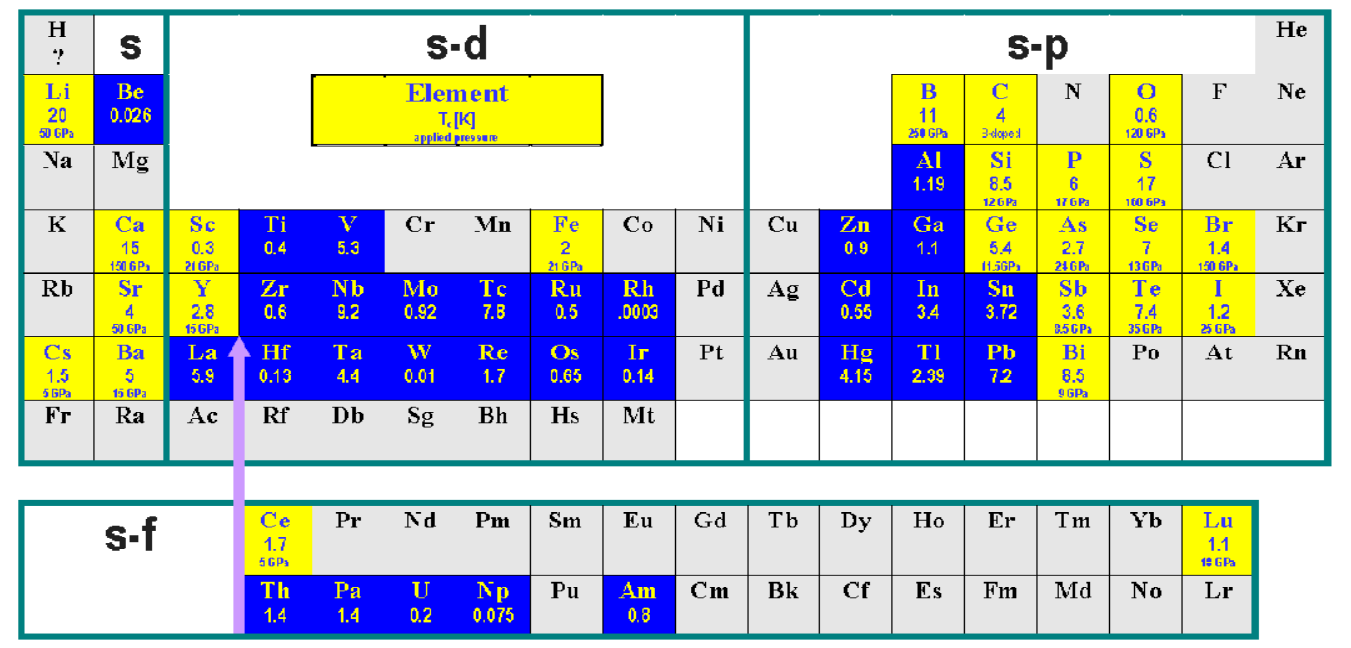

Fig. 1. Periodic table with the distribution and $T_{c}[K]$ of the chemical elements for which superconductivity has been observed with or without application of pressure. ${ }^{[1,6,7,8]}$

From the discovery of superconductor many scientist has tried to establish a theoretical phenomenon of how a material behave like a superconductor when it is cooled below its characteristics critical temperature. As a result in 1957, Bardeen, Cooper, and Schrieffer developed such a theory called the BCS theory ${ }^{9}$. The BCS theory is a successful theory in condensed-matter physics, since a rigorous treatment of electron-phonon interaction is applicable using the many-body perturbation theory ${ }^{10}$. However, the BCS theory is inapplicable in unconventional superconductors, where electron-electron interaction plays an important role in electron pairing instead of electron-phonon interaction. We are required to develop a different theory that rigorously explains the unconventional superconductors. It is one of the biggest unsolved problems in theoretical physics yet. Recently, however, scientists at Chinese Academy of Sciences, Beijing and the Santa Fe Institute proposed such a model in the form of an experimentbased phenomenological BCS-like expression for the superconducting transition temperature $T_{c}$ in heavy-electron materials that is based on a simple model for the effective range and strength of the spin-fluctuation-induced quasiparticle interaction, and reflects the unusual properties of the heavy-electron normal state - in which electrons gain mass as local electron spins lose their magnetism - from which superconductivity emerges ${ }^{11}$. For proper application of a superconductor in practical we need to discover a superconducting material which will be able to show its superconducting properties in room temperature. Though for getting a superconducting state a material is cooled below its critical temperature, recently a group of researchers have succeed for the first time in making a ceramic superconducting at room temperature with the aid of short infrared laser pulses - albeit for only a few millionths of a microsecond ${ }^{12}$. As surprising characteristics of high temperature superconductors have been revealed, application has accelerated in the last ten years. Developments include high-quality bulks, high-quality thin films and high-quality superconducting wires and tapes. These will be used in electric communication systems, superconducting power cables, superconducting magnetic energy storage systems, electric motors and many other types of superconducting magnets.

\section{Conventional Superconductor}

A superconductor is generally considered as a conventional superconductor if it can be explained by BCS theory. Conventional superconductors can be either type-I or type-II. Most of the elemental superconductors are conventional. Niobium and vanadium are type-II, while most other elemental superconductors are type-I. In1911, Kamerlingh Onnes first discovered the phenomenon of superconductivity in mercury ${ }^{13}$ by an experimental error, such as an accidental short circuit. Later on careful repetition assured him that he had in fact discovered a new electronic phase. The discovery of vanishing resistivity in several other elements such as tin and lead soon followed. The second characteristic of superconductivity, expulsion of magnetic flux from the superconducting state, was discovered by Meissner and Ochsenfeld in $1933^{14}$. Major 
advances were made with the London theory ${ }^{15}$ in 1935 and the Ginzburg-Landau theory ${ }^{16}$ in 1950. A universally accepted microscopic theory of the phenomenon was put forth by Bardeen, Cooper, and Schrieffer (BCS) in 1957. ${ }^{17}$ They ascribed superconductivity to an attractive interaction between two electrons through electron-phonon interaction in BCS theory of superconductivity. One electron interacts with a positive ion in the lattice and deforms the lattice; a second electron of compatible momentum value interacts with the ion in the distorted lattice so as to minimize the energy. The interacting electrons are said to form a pair. The electrons of a pair have equal and opposite momentum; one in spin up state and the other in spin down state, so that the total spin of a pair is zero. The electron pairs are called Cooper pairs. The important feature of Cooper pair is that excitation can take place in only pairs of electrons; i.e., if the state with $+\mathrm{k}$ and spin up ( $\uparrow)$ is occupied, the corresponding state with $-\mathrm{k}$ and spin down $(\downarrow)$ is also occupied and if the state with $+\mathrm{k}$ is vacant, the state with $-\mathrm{k}$ is also vacant. Two of the main experimental facts that led to the BCS understanding were:

(a) The density of states is gapped at the Fermi surface. This was determined experimentally first by the measurement of an exponential specific heat. ${ }^{[18,19]}$ This led to the realization that some kind of pairing is occurring and

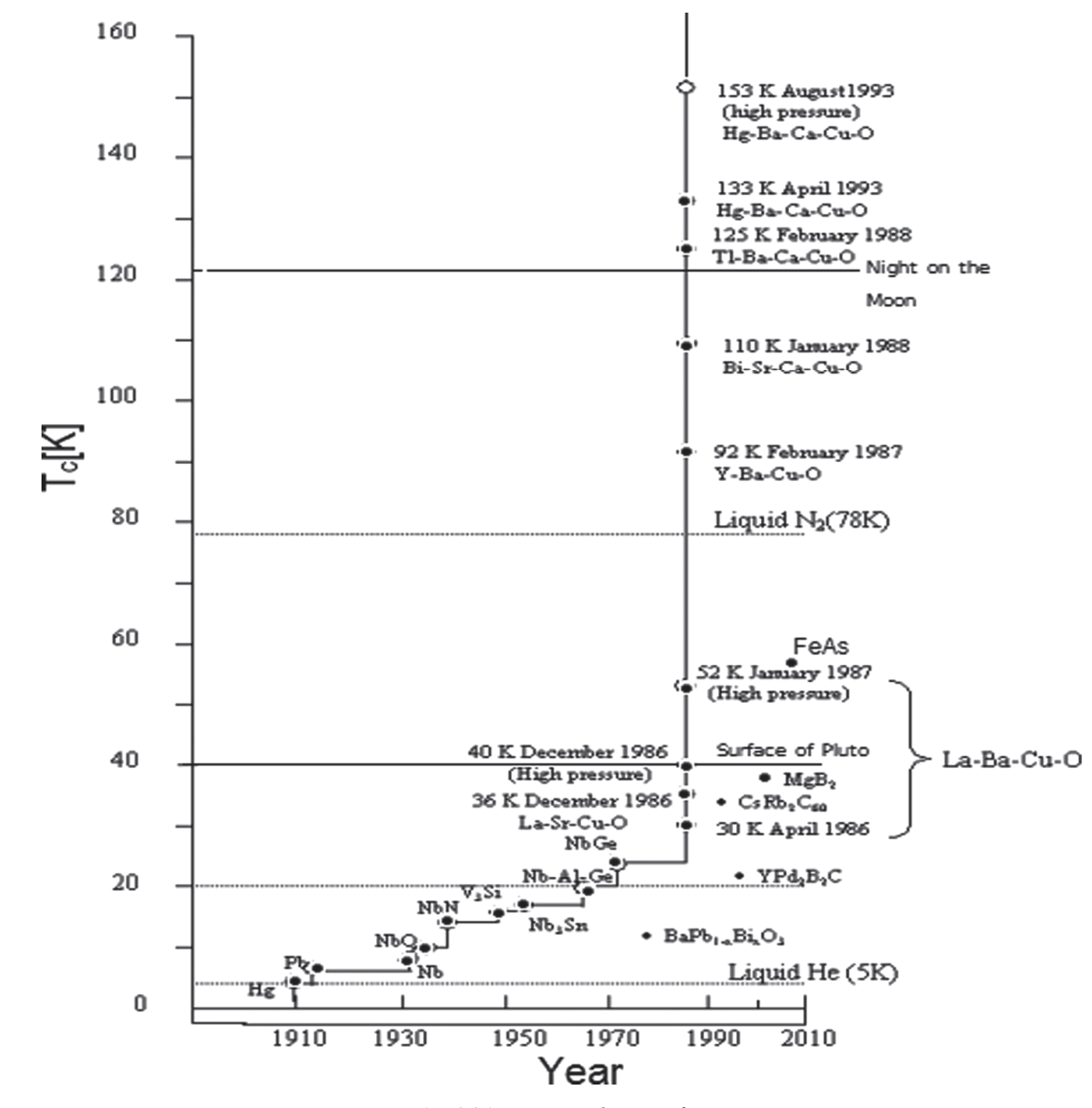

Fig. 2(a). Time evolution of $T_{c}$.

In 1986, a revolution occurs in the field of superconducting research. In this year Bednorz and Muller, working at IBM in electrons are thermally activated across a gap with Boltzmann probability. The gap was later confirmed by electromagnetic absorption in aluminum and lead. ${ }^{[20-22]}$

(b) The $T_{c}$ was found to vary as the inverse square root of the nuclear mass, so the phonons concern was shown experimentally by the isotope effect. ${ }^{[23,24]}$ Since the phonon frequency varies as $\sqrt{\mathrm{k}} / \mathrm{M}$, the discovery of the isotope effect led to the understanding that phonons are involved.

Numerous details were filled in over the next few decades, but the problem of superconductivity was largely considered solved by BCS in 1957.

\section{Unconventional Superconductor}

A superconductor is considered as an unconventional superconductor if it can't be explained by BCS theory. The high temperature superconductors generally considered as unconventional superconductors since it is not explained by BCS theory. Some examples of unconventional superconductors are $\mathrm{CeCu}_{2} \mathrm{Si}_{2}$, (TMTSF) ${ }_{2 \mathrm{PF} 6}$ and $\mathrm{LaOFeAs}$. High-temperature superconductors are materials that behave as superconductor at unusually high temperatures. A superconductor is generally considered as high temperature superconductor if it's $T_{c}$ is greater than $77 \mathrm{k}$ (i.e, $T_{c}>77 \mathrm{~K}$ ).

Switzerland, discovered a new class of superconducting materials having $T_{c}$ up to $30 \mathrm{k}^{25}$ Whereas ordinary of 
conventional superconductors usually have transition temperatures below 30k HTS have been observed with transition temperatures as high as $138 \mathrm{k}$. A history of the increase in record $T_{c}$ is shown in Figure 2(a). Though the transition temperature of superconductors have been gradually increased day by day and consequently the hightemperature superconductors have been discovered, but till this moment there is no satisfied theory by which the hightemperature superconductivity can be explained. Where in conventional superconductors, simple s-wave gap is found, in case of high-temperature superconductors it is generally accepted that there is a $d x^{2}-y^{2}$ wave gap in the density of states. This was shown by flux modulation measurements in YBCO DC-SQUID ${ }^{26}$ and then more explicitly by flux quantization in a tri-crystal YBCO junction. ${ }^{27}$ From the gap in the density of states, it is usually decided that electrons are paired. By the measurement of $h / 2 e$ flux quanta, ${ }^{28}$ it was shown explicitly that the charge carriers have charge $2 e$. At low carrier concentration, magnetism is known to play a role in the crystal, so many arguments strongly support the point that electron pairing in HTSC is caused by magnons or other magnetic consequences. But the pairing is indeed caused by phonons have also carried strong arguments. ${ }^{29}$

\section{Cuprate Superconductors}

In more than 24 years elapsed from the discovery of high $\mathrm{T}_{\mathrm{c}}$ superconductivity in copper oxides, a huge number of experimental and theoretical investigations of the physical properties of these materials have been done. The hightemperature superconductivity of cuprates was discovered in 1986, when the highest superconducting transition temperature (i.e., critical temperature) characteristic of conventional superconductors $\left(T_{c}=23.2 \mathrm{~K}\right.$ in $\left.\mathrm{Nb}_{3} \mathrm{Ge}\right)$ was substantially exceeded and a superconducting transition temperature $T_{c}=30 \mathrm{~K}$ was achieved in the ceramic $\mathrm{La}_{2}$ ${ }_{\mathrm{x}} \mathrm{Ba}_{\mathrm{x}} \mathrm{CuO}_{4-\delta}$. The situation including the high $T_{c}$ cuprate superconductors is illustrated in Fig. 2(b). Within a year after this discovery, the record value of $T_{c}$ exceeded $90 \mathrm{~K}$ ( $\mathrm{YBa}_{2} \mathrm{Cu}_{3} \mathrm{O}_{7-\delta}$ ceramic). The further search for and creation of new superconducting materials led to $T_{c}=138 \mathrm{~K}$ (Tl-doped $\mathrm{HgBa}_{2} \mathrm{Ca}_{2} \mathrm{Cu}_{3} \mathrm{O}_{8-} \delta$ compound) in 1994 and raised the question of room-temperature superconductivity. In spite of all these efforts, the mechanism of this new kind of superconductivity has not been clarified yet; it still remains one of the most enigmatic problems of the solid-state physics. The description of their normal state properties has turned out to represent an even bigger challenge to solid-state physics theory. The difficulty of this problem is due to the complicated properties, including complicated crystalline structures of materials displaying high $T_{c}$, to the presence of a strong anisotropy, to the existence of non-adiabatic effects, to strong electronic correlations, and to a strong electronphonon interaction. In these complicated materials, several phase transitions (structural, magnetic, superconductor, etc.) occur, and mixed states are allowed, for instance coexistence of superconductivity and ferromagnetism or vitreous spin state. The key structural element of layered quasi-twodimensional cuprates is a $\left(\mathrm{CuO}_{2}\right)$ plane (one or several in a unit cell); they differ from conventional superconductors not only in high values of $T_{c}$ but also in a set of physical properties that cannot be described by the classical BardeenCooper-Schriefffer (BCS) scheme. In cuprates, charge carriers appear due to the doping of the $\mathrm{CuO}_{2}$ planes of a parent antiferromagnetic insulator upon nonisovalent atomic substitution or the creation of oxygen vacancies in charge reservoirs outside the conducting planes. The distance between equivalent $\mathrm{CuO}_{2}$ planes in neighboring unit cells is large compared to the in-plane distance between neighboring copper atoms, which results in a strong conductivity anisotropy at temperatures above $T_{c}$ and the two-dimensional coherence of the superconducting state at temperatures below $T_{c}$. The highlighting feature of this type of superconductors that is more attractive for application point of view is the very high upper critical field. Before discussing the characteristics and application of cuprate family we will discuss about some basic properties of superconductors. Because these properties are also present in cuprate superconductors.

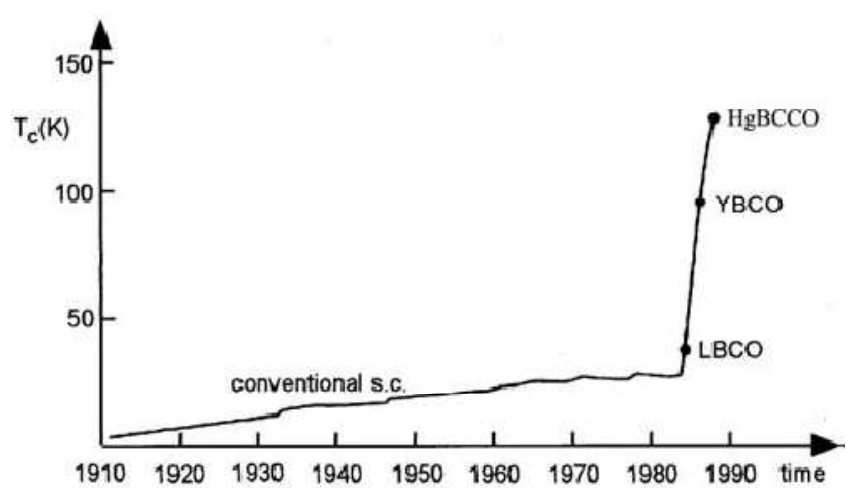

Fig. 2(b). High- $T_{c}$ cuprate superconductors discovered by Bednorz and Mueller in 1986. For $\mathrm{La}_{2-x} \mathrm{Ba}_{x} \mathrm{CuO}_{4}$ a $\mathrm{T}_{c} \approx 35 \mathrm{~K}$, for $\mathrm{YBa}_{2} \mathrm{Cu}_{3} \mathrm{O}_{7-\delta}$ a $\mathrm{T}_{c}=92 \mathrm{~K}$, and for $\mathrm{HgBa}_{2} \mathrm{Ca}_{2} \mathrm{Cu}_{3} \mathrm{O}_{8+\delta}$ a $\mathrm{T}_{c}=133 \mathrm{~K}$ was observed, for example.

\section{The Basic Properties of Superconductors}

A superconductor has several main macroscopic characteristics, such as

1. Zero Resistance.

2. The Meissner Effect.

3. The Josephson Effect.

4. The Isotope Effect.

5. Anomalous Specific Heat Capacity and

6. Abnormal Infrared Electromagnetic Absorption.

Here we will focus on three basic properties, namely zero resistance, the Meissner effect and the Josephson Effect.

\subsection{Zero-Resistance Characteristics}

The zero resistance characteristic of the superconductor refers to the phenomenon that resistance abruptly disappears at a certain temperature. It is able to transport 
direct current (DC) without resistance in the superconducting state. If a closed loop is formed by a superconductor in which a current is induced, the induced "persistent current" will show no obvious signs of decay for several years. The upper limit of resistivity measured by the "persistent current" experiment is less than $10^{-27} \Omega-\mathrm{m}$, while a good conventional conductor, such as copper, has a resistivity of $10^{-10} \Omega-\mathrm{m}$ at $4.2 \mathrm{~K}$, which is more than 17 orders of magnitude than that of the superconductor. The typically experimental dependence of resistance on temperature in a superconductor is shown in Figure 3, in which the resistivity of the superconductor suddenly falls to zero when the temperature reduces to a certain value below the critical temperature $T_{\mathrm{c}}$. The zero resistance property of a superconductor is not the minimal resistance in the usual sense but is equal to zero. This is because carriers are not scattered by the crystal lattice, thus there is no energy dissipation in a superconductor carrying a DC current, which suggests that superconductivity is a kind of macroscopic quantum effect. Since the discovery of superconductivity in 1911, there have been many attempts to establish a theory to explain this phenomenon, and a number of models describing physical characteristics of superconductors have been established. Some simple and easily understandable models belong to phenomenological theories of which the two-fluid model is a relatively intuitive theory. This model can successfully describe motion of carriers and magnetic field distribution within the superconductor. Combined with the constitutive Maxwell's electromagnetism equations, the two-fluid model explains some superconducting phenomena such as zero resistance and the Meissner effect. Based on a series of interaction hypotheses between electrons and lattice in quantum mechanics, in 1957, J. Bardeen, L.N. Cooper and J.R. Schrieffer proposed the concept of Cooper pairs and established the well-known Barden-Cooper-Schrieffer (BCS) theory, that is, the superconducting quantum theory that describes superconductivity from the microscopic point of view and successfully explains most of superconducting phenomena.

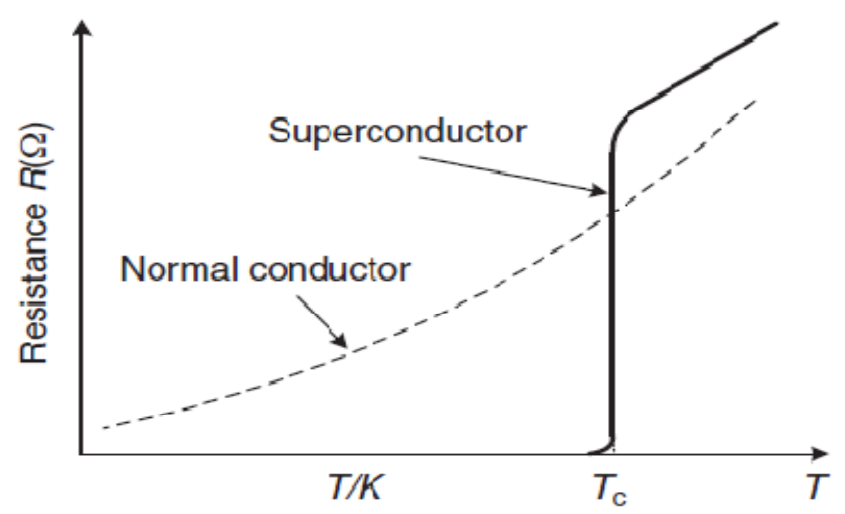

Fig. 3. Resistance versus temperature curve of superconductors and normal conductors.

\subsection{Complete Diamagnetism - Meissner Effect}

When the superconductor is subjected to a magnetic field, in a non-superconducting state, the magnetic field can penetrate the superconductor and so the inner magnetic field is not zero in its normal state (Fig. 4(a)). ${ }^{30}$ However, when the superconductor is in a superconducting state, the magnetic flux within is completely excluded from the superconductor, and the inner magnetic field is zero, that is, the superconductor is completely diamagnetic (Fig. 4(b)). This phenomenon is called the Meissner effect. The superconductor can be suspended in a magnetic field due to its diamagnetism or the Meissner effect (Fig. 4(b)), in which a YBCO bulk is in a magnetic field produced by conventional magnetic materials (NdFeB permanent). When the YBCO bulk is in a superconducting state, with liquid nitrogen temperature of $77 \mathrm{~K}$, it is suspended in air because the magnetic flux is completely excluded from the YBCO, which results in a magnetic levitation force.

\subsection{Josephson Effects}

As with semiconductor and thermocouple devices, there is the concept of a junction in a superconductor when it joins with an insulator. Two superconductors are separated by a thin insulating layer whose thickness is so small that Cooper pairs can pass through by the tunneling effect. ${ }^{[31,32]}$ This geometry is called the Josephson junction (Fig. 5).

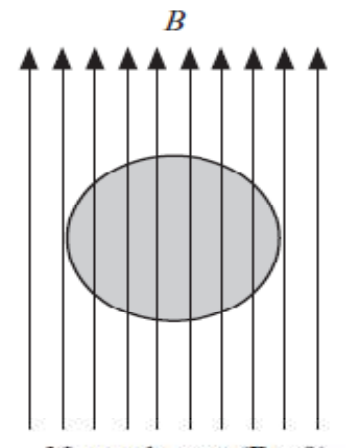

Normal state $(B \neq 0)$

(a)

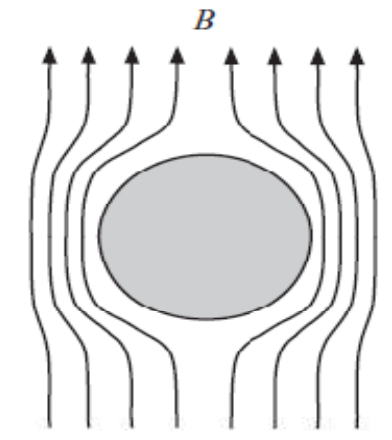

Superconducting state $(B=0)$

(b)

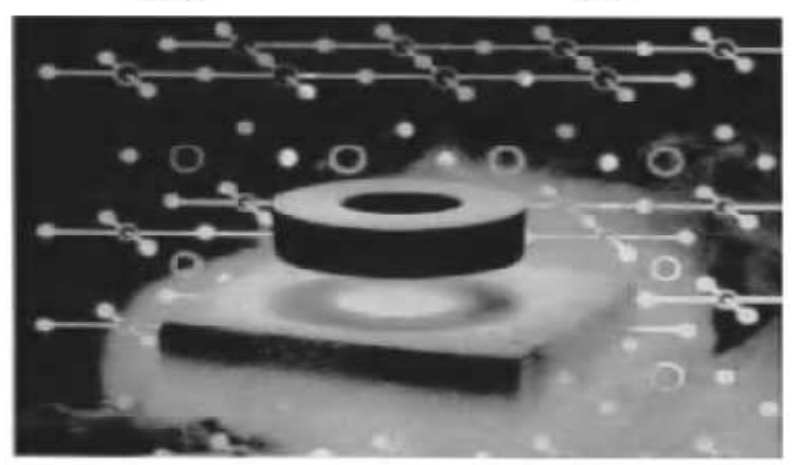

(c)

Fig. 4. Meissner effect and levitation of superconductor: (a) normal state; (b) Meissner state; (c) YBCO bulk levitation. 


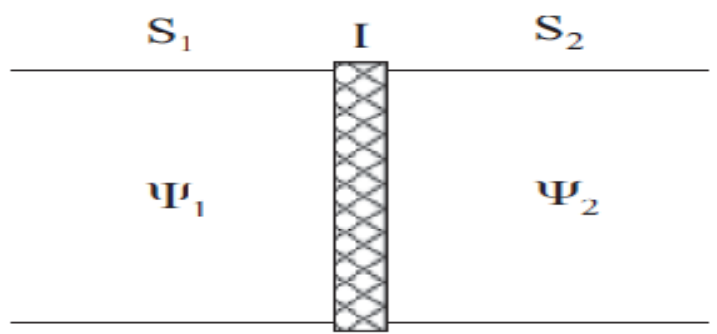

Fig. 5. Geometry of Josephson junction (here, $s_{1}$ and $s_{2}$ two kind of superconductor, I is thin insulating layer).

The Josephson Effect is the phenomenon of supercurrent (A current that flows indefinitely long without any voltage applied) across a device which is known as Josephson junction. The Josephson Effect is an important basis of superconducting electronics applications and has widespread applications in many instruments such as voltage reference, superconducting cavities, superconducting filters and SQUIDs (If two Josephson junctions are connected in parallel, a loop is formed, known as the superconducting quantum interference device (SQUID)). For example, it is the most sensitive method to detect a magnetic field, by using SQUID to measure current oscillations. SQUID can convert many other small signals in the magnetic field, so it can also be employed to measure these small current signals in clinical diagnosis, such as the magnetocardiogram (MCG) and the magnetoencephalogram (MEG). Superconducting tunnelling junctions can also be used as basic devices for high-speed, low-dissipation logic families.

\section{Common Characteristics of Cuprate Superconductors}

After invention of high-temperature superconductivity in cuprate based material many characteristics of these material has been invented. Among all of these characteristics some are common in all cuprate family such as crystal structure, electronic structure etc. In this section some common characteristics will be discussed. The specific characteristics will be discussed in later sections.

\subsection{The Crystal Structure and Classification of Cuprates}

A very common characteristic of cuprate superconductors is that they are divided into two parts, (a) $\mathrm{CuO}_{2}$ planes and (b) Blocking layers (Fig. 6). Superconductivity occurs in only $\mathrm{CuO}_{2}$ planes and the blocking layers supply charge carriers to the $\mathrm{CuO}_{2}$ planes. ${ }^{33}$

All cuprate superconductors have a general Perovskite Structure. Perovskites are ceramics (solid materials combining metallic elements with non-metals, usually oxygen) that have a particular atomic arrangement. In their ideal form, described by generalized formula $\mathrm{ABX}_{3}$, they consist of cubes made up of three distinct chemical elements $(\mathrm{A}, \mathrm{B}$ and $\mathrm{X}$ ) that are present in ratio $1: 1: 3$. The $\mathrm{A}$ and $\mathrm{B}$ atoms are metallic cations (positive) and $\mathrm{X}$ atoms are nonmetallic anions (negative). An A cation - the largest of the two kinds of metals - lies at the centre of each cube, the B cations occupy all the eight corners and the $\mathrm{X}$ anions lies at the mid-points of cubes twelve edges (Fig. 7). $\mathrm{CuO}_{2}$ planes plays a crucial role in superconducting in cuprate compounds As shown in Table 1 crystal structure of cuprate compounds basically is tetragonal. Highest critical temperature reached in cuprates having flat and square $\mathrm{CuO}_{2}$ planes. The $\mathrm{CuO}_{2}$ planes in cuprates separated by the atoms as $\mathrm{Bi}, \mathrm{O}, \mathrm{Y}, \mathrm{Ba}, \mathrm{La}$, etc. which plays role of charge reservoirs.

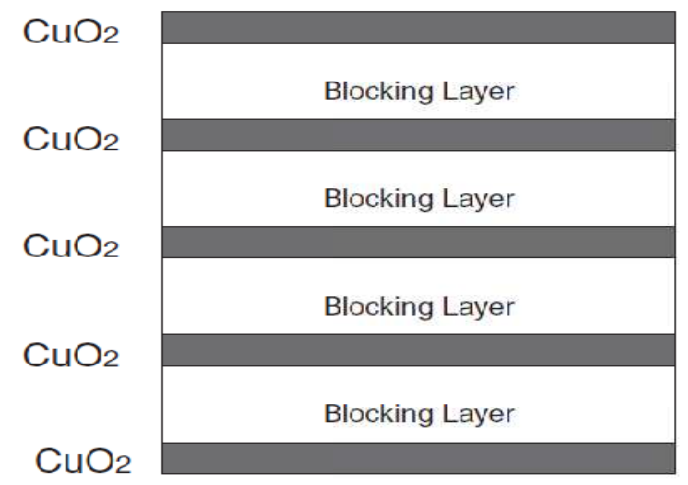

Fig. 6. Schematic structure of high-temperature superconductors.

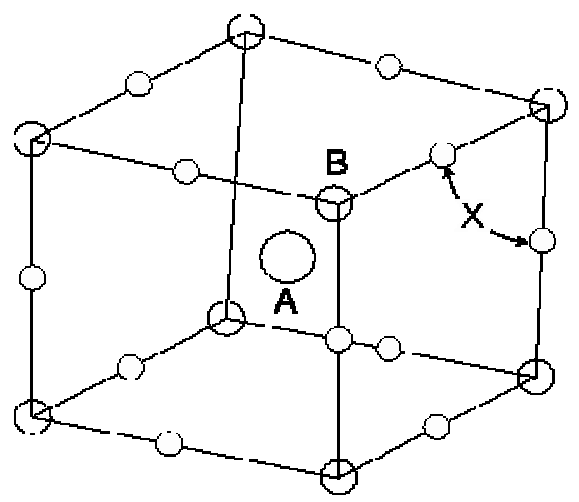

Fig. 7. The perovskite structure $A B X_{3}$.

Table 1. Crystal structure and elementary cell of some cuprates

\begin{tabular}{llll}
\hline Compounds & Crystal Structure & Size of elementary cell, & $\boldsymbol{T}_{\boldsymbol{c}}, \mathbf{K}$ \\
\hline $\mathrm{La}_{2-\mathrm{x}} \mathrm{Sr}_{\mathrm{x}} \mathrm{C} u \mathrm{O}_{4}(\mathrm{La}=\mathrm{Sr}-214)$ & Tetragonal & $\mathrm{a}=\mathrm{b}=3: 78 ; \mathrm{c}=13.2$ \\
$\mathrm{YBa}_{2} \mathrm{C} u_{3} \mathrm{O}_{7}(123)$ & Orthorhombic & $\mathrm{a}=3.82 ; \mathrm{b}=3.88 ; \mathrm{c}=11.7$ \\
$\mathrm{BiSr}_{2} \mathrm{CaCu}_{2} \mathrm{O}_{8}(\mathrm{Bi}-2212)$ & Tetragonal & $\mathrm{a}=\mathrm{b}=5.4 ; \mathrm{c}=30.89$ \\
$\mathrm{~T} \mathrm{Ba}_{2} \mathrm{Ca}_{2} \mathrm{Cu}_{3} \mathrm{O}_{9}(\mathrm{~T} 1-1223)$ & Tetragonal & $\mathrm{a}=\mathrm{b}=3.85 ; \mathrm{c}=15.9$ & 90 \\
$\mathrm{~T}_{2} \mathrm{Ba}_{2} \mathrm{C} u \mathrm{O}_{6}(\mathrm{~T} 1-2201)$ & Orthorhombic & $\mathrm{a}=5.468 ; \mathrm{b}=5.473 ; \mathrm{c}=23.24$ \\
$\mathrm{~T}_{2} \mathrm{Ba}_{2} \mathrm{CaCu}_{2} \mathrm{O}_{8}(\mathrm{~T} 1-2212)$ & Tetragonal & $\mathrm{a}=\mathrm{b}=3.86 ; \mathrm{c}=29.3$ \\
$\mathrm{~T}_{2} \mathrm{Ba}_{2} \mathrm{Ca}_{2} \mathrm{Cu}_{3} \mathrm{O}_{10}(\mathrm{~T} 1-2223)$ & Tetragonal & $\mathrm{a}=\mathrm{b}=3.85 ; \mathrm{c}=35.9$ \\
$\mathrm{HgBa}_{2} \mathrm{Ca}_{2} \mathrm{Cu}_{3} \mathrm{O}_{8}(\mathrm{Hg}-1223)$ & Tetragonal & $\mathrm{a}=\mathrm{b}=3.85 ; \mathrm{c}=15.9$ \\
$\mathrm{HgBa}_{2} \mathrm{Ca}_{3} \mathrm{Cu}_{4} \mathrm{O}_{10}(\mathrm{Hg}-1234)$ & Tetragonal & $\mathrm{a}=\mathrm{b}=3.85 ; \mathrm{c}=19$ \\
\hline
\end{tabular}




\subsection{The Phase Diagram of Cuprates}

A typical phase diagram of cuprate high-temperature superconductor is shown in Fig. 8, in which the phase of zero dopant concentration is antiferromagnetic (AF), and the doped carriers (electrons or holes) destroy the antiferromagnetic phase and induce superconductivity (SC). Hole doped cuprates present superconductivity when doping level is around $0.05<x<0.27$ where $x$ is the hole doping per $\mathrm{Cu}$ in $\mathrm{Cu}-\mathrm{O}$ planes. The doping level with $x=0.16$ is optimal doping of which the $T_{c}$ is maximum, and the doping level with $x<0.16, x>0.16$ are underdoped region, overdoped region respectively. It is well-known that the superconducting critical temperature, $T_{c}$, for all cuprates approximatively fulfils a universal equation $T_{c} / T_{c \max }=1-$ $82.6(x-0.16)^{2}$ in which $T_{c} \max$ is different for different families. ${ }^{[34,35]}$ The $T_{c}$ as a function of doping level is shown in Fig-8. Around optimally doping level, the normal state resistivity of sample presents perfect linearity as a function of temperature, and the temperature could be extended from low critical temperature $\left(T_{c}\right)$ to very high up critical temperature (e.g., the up critical temperature could be as high as $1000 \mathrm{~K}$ in La-214 system) ${ }^{36}$ which is obviously deviated from the square relationship in Fermi liquid theory. In underdoped region, sample shows strange normal state that couldn't be described by conventional Fermi-Landau liquid theory which works quite well in the metals. For underdoped sample, an energy gap is opened above superconducting transition temperature $T_{c}{ }^{[37-39]}$, and this gap is called pseudogap, while the energy gap is only opened below $T_{c}$ in conventional superconductors. It's believed that clarifying the physics in underdoped region is indispensable in understanding the superconducting mechanism of cuprates, and whether the pseudogap state above $T_{c}$ is a competitive phase of superconductivity or a precursor of superconductivity. In the normal state of overdoped region and the nonsuperconducting region with doping level $x>0.25$, the material presents characteristic of normal metal. For electrondoped region, the material presents antiferromagnetic in a wide doping region which is shown in Fig. 8, and only presents superconductivity in a narrow doping region. In addition, there are only limited kinds of electrondoped materials, so the underdoped region is less studied than the overdoped region. The Bismuth families are of the most popular materials in cuprates, and their genericchemical formula is $\mathrm{Bi}_{2} \mathrm{Sr}_{2} \mathrm{Ca}_{n} \mathrm{Cu}_{n+1} \mathrm{O}_{2 n+6+\delta}$. Depending on different layers of $\mathrm{Cu}-\mathrm{O}$ plane, there are three kinds of materials including $\mathrm{Bi}_{2} \mathrm{Sr}_{2} \mathrm{CuO}_{6+\delta}(\mathrm{Bi} 220, n=1){ }^{40}, \mathrm{Bi}_{2} \mathrm{Sr}_{2} \mathrm{Ca}_{1 C_{2}} \mathrm{O}_{8}+\delta$ $(\mathrm{Bi} 2212, n=2){ }^{41}$ and $\mathrm{Bi}_{2} \mathrm{Sr}_{2} \mathrm{Ca}_{2} \mathrm{Cu}_{3} \mathrm{O}_{10+\delta}(\mathrm{Bi} 2223, n=3)^{41}$. In the past twenty years, most of photoemission results were from studying on Bi2212. Because of its easy cleaving, high superconducting transition temperature and wide doping range, Bi2212 is one of the most optimal samples in photoemission experiments.

\subsection{Electronics Structure of Cuprates}

A common structure of cuprate superconductors is the $\mathrm{Cu}$ $\mathrm{O}$ plane which is in charge of their intrinsic electronic structures. In the form of $\mathrm{ABO}_{3}$ structure, $\mathrm{Cu}^{2+}$ is surrounded by four $\mathrm{O}^{2-}$ and apical oxygen(s) or halogen(s) perpendicular to the plane. Taking $\mathrm{La}_{2} \mathrm{SrCuO}_{4}$ for an example, theCu${ }^{2+}$ corresponds to $3 \mathrm{~d}_{9}$ electronic configuration, and while in crystal field, the $3 \mathrm{~d}_{9}$ orbital is splitted into five d-orbitals, as shown in Fig. 9. ${ }^{42}$ Four orbitals with lower energy including $x y, x z, y z$ and $3 z^{2}-r^{2}$ are fully occupied, while the orbital $x^{2}-$ $y^{2}$ of highest energy is half filled. Because of parallel energy of the $\mathrm{Cu}$ d-orbitals and the $\mathrm{O} 2 \mathrm{p}$-orbitals, there is a strong hybridization between them. As a result, the topmost energy level involves the feature of both $\mathrm{Cu} \mathrm{d}^{2}-y^{2}$ and $\mathrm{O} 2 \mathrm{p}_{x, y}$ orbitals.

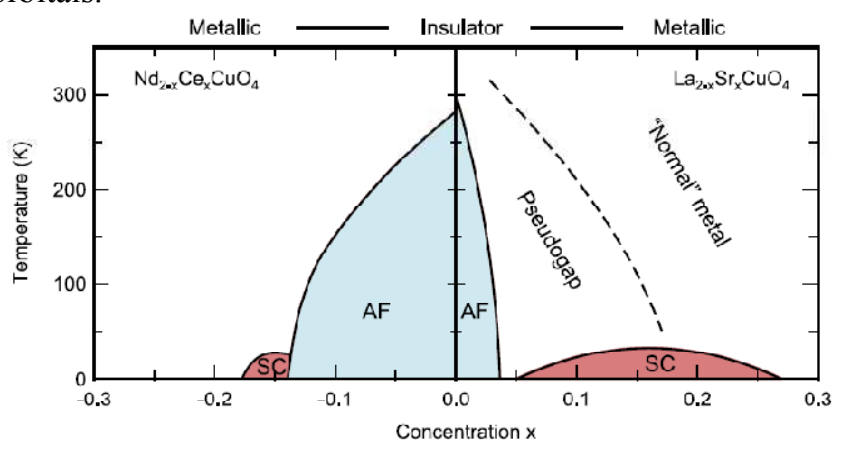

Fig. 8. Phase diagram of electron- and hole-doped cuprates, showing superconductivity (SC), antiferromagnetic (AF), pseudogap, and normalmetal regions.

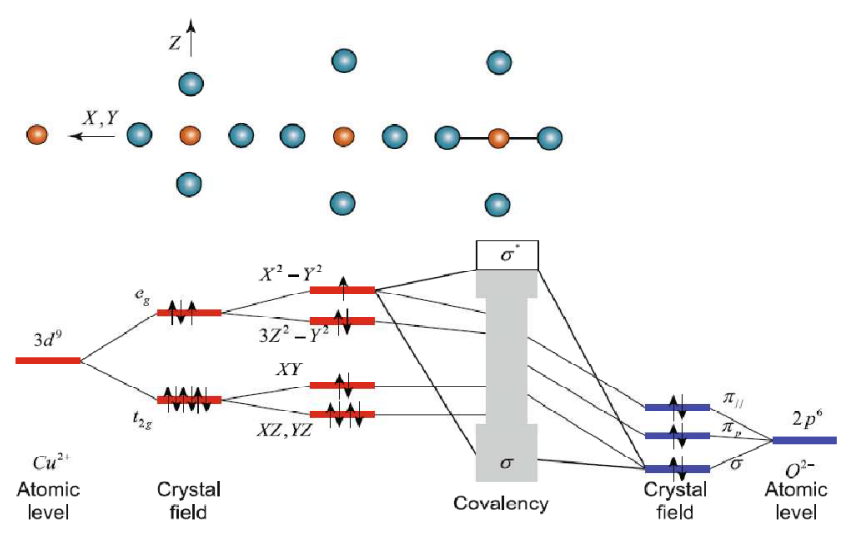

Fig. 9. Bonding in $\mathrm{Cu}$-O plane.

\section{Specific Characteristics of Cuprates Depending Upon Different Compound}

In this section all properties and characteristics of different cuprate based compound will be discussed. The applications of those compounds will also be discussed.

\section{1. $\mathrm{La}_{2-x} \mathrm{M}_{x} \mathrm{CuO}_{4}$ Superconductors}

In 1986 Bednorz and Muller first discovered the lanthanum family of HTSC. It was the first family of high- 
temperature superconductors. This family of superconductors has tetragonal crystal structure. The size of elementary cell is $\mathrm{a}=\mathrm{b}=3.78, \mathrm{c}=13.2$. The highest $T_{c}$ of this family is achieved $37.5 \mathrm{k}$. Synthesis of alkaline earth doped $\mathrm{La}_{2-}$ ${ }_{\mathrm{x}} \mathrm{M}_{\mathrm{x}} \mathrm{CuO}_{4}(\mathrm{M}=\mathrm{Ca}, \mathrm{Sr}, \mathrm{Ba})$ of $\mathrm{K}_{2} \mathrm{NiF}_{4}$ structure with $T_{c}$ up to $35 \mathrm{k}$ is achieved by ceramic method. This cuprates have structure derived from ideal perovskite structure through an intergrowth phenomenon. Structure of $\mathrm{La}_{2-\mathrm{x}} \mathrm{M}_{\mathrm{x}} \mathrm{CuO}_{4}$ is shown in Fig. 10. $\mathrm{M}$ is substituted on the La-site. $\mathrm{La}_{1.85} \mathrm{Sr}_{0.15} \mathrm{CuO}_{4}$

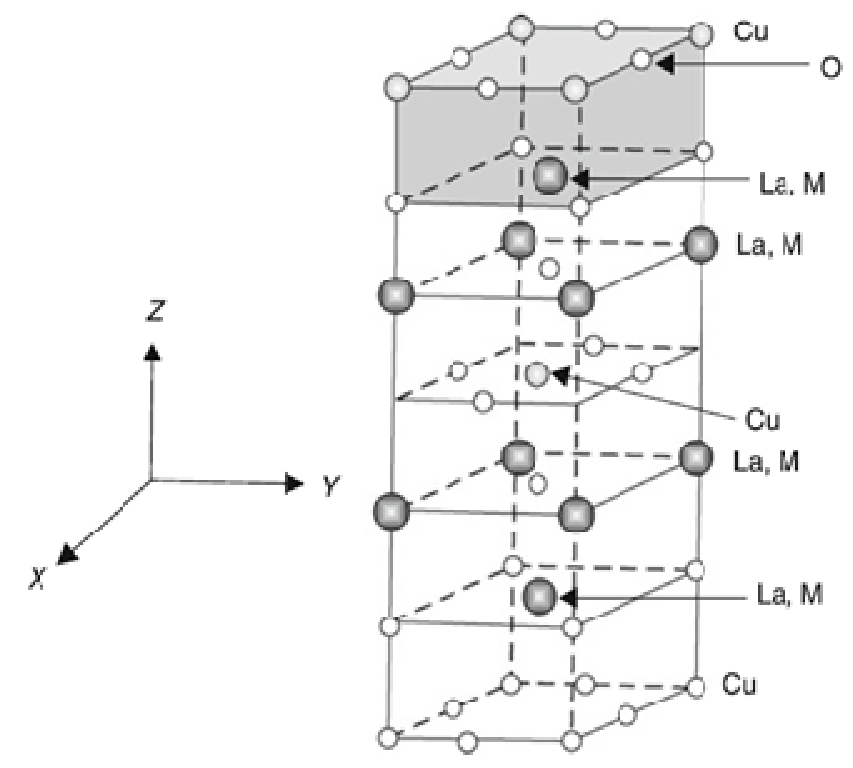

has a tetragonal $\mathrm{K}_{2} \mathrm{NiF}_{4}$ type of layered perovskite structure. The structure can be viewed as stacking of

$$
-\mathrm{CuO}_{2}-\mathrm{LaO}-\mathrm{LaO}-\mathrm{CuO}_{2}-\mathrm{LaO}-\mathrm{LaO}-\mathrm{CuO}_{2}-
$$

$\mathrm{La}_{2-\mathrm{x}} \mathrm{M}_{\mathrm{x}} \mathrm{CuO}_{4}$ shows the highest $T_{c}$ around a critical value of $\mathrm{x}\left(0.15\right.$ for $\mathrm{Sr}$ and 0.2 for $\mathrm{Ba}$ with $T_{c}$ of 30 and $35 \mathrm{k}$ respectively).

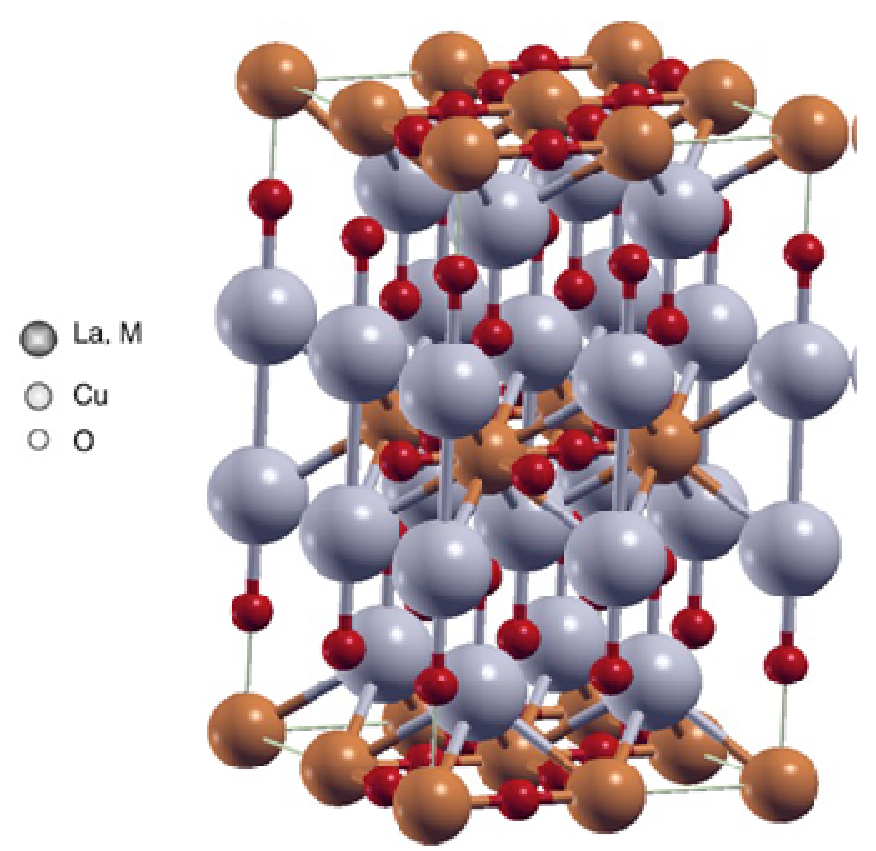

Fig. 10. The crystal structure of $\mathrm{La}_{2-x} \mathrm{M}_{x} \mathrm{CuO}_{4}\left(\mathrm{M}=\mathrm{Ba}\right.$ or Sr). For $x=0, a=b=3.77 \AA$, c $=13.25$ A. M atoms occupy La site in La $\mathrm{CuO}_{4}$.

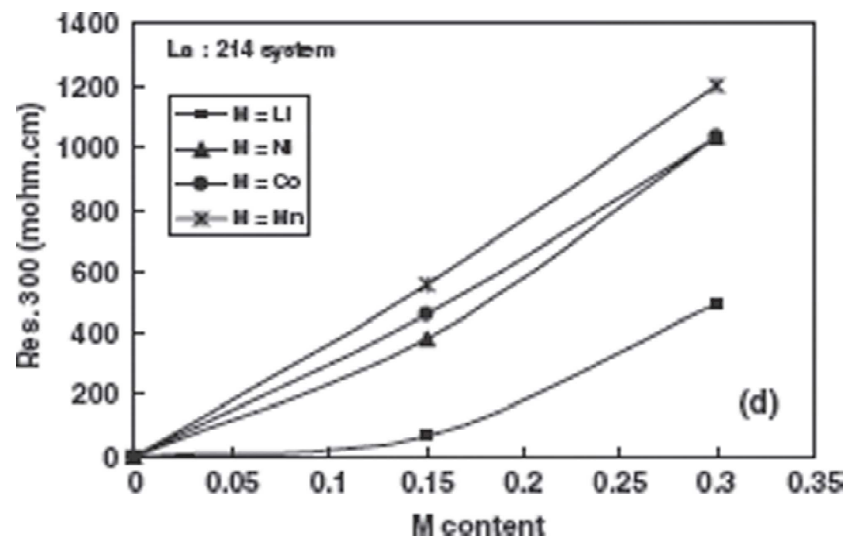

Fig. 11. Normal state resistivity versus doping content for $\mathrm{La}_{1.85} \mathrm{Sr}_{0.15} \mathrm{Cu}$. ${ }_{x} M_{x} O_{y}$ ceramic samples.

Sedky et al. ${ }^{43}$ investigated the structural and transport properties of $\mathrm{La}_{1.85} \mathrm{Sr}_{0.15} \mathrm{Cu}_{1-\mathrm{x}} \mathrm{M}_{\mathrm{x}} \mathrm{O}_{\mathrm{y}}(\mathrm{M}=\mathrm{Li}, \mathrm{Ni}, \mathrm{Co}, \mathrm{Mn}$, and $\mathrm{x}=0.00,0.15,0.30)$ ceramic samples. They showed that the magnetic doping elements caused lattice deformation resulting in fast increase in the resistivity more efficiently than the non-magnetic doped samples and direct to the largest change in the transport properties of La-214 systems. Normal state resistivity versus doping content for $\mathrm{La}_{1.85} \mathrm{Sr}_{0.15} \mathrm{Cu}_{1 \text { - }}$ ${ }_{x} \mathrm{M}_{\mathrm{x}} \mathrm{O}_{\mathrm{y}}$ ceramic samples is shown in Fig. 11.

\subsection{YВаCИO Superconductors}

M.K. Wu and his graduate students, Ashburn and Torng ${ }^{44}$ at the University of Houston in 1987 discovered YBCO has a critical temperature of $93 \mathrm{~K}$. $\mathrm{YBaCuO}$ (Yttrium Barium Copper oxide) is the first material to become superconducting above $77 \mathrm{k}$, the boiling point of liquid nitrogen. It is a family of crystalline chemical compounds. The general formula of the family is $\mathrm{YBa}_{2} \mathrm{Cu}_{3} \mathrm{O}_{7-\mathrm{x}}$. The compound $\mathrm{YBa}_{2} \mathrm{Cu}_{3} \mathrm{O}_{7}$ is superconducting $\left(T_{\mathrm{c}}=90 \mathrm{~K}\right)$ and has orthorhombic symmetry and, the structure has two $\mathrm{Cu}-\mathrm{O}$ sheets in the $a b$ plane and $\mathrm{Cu}-\mathrm{O}$ chains along the $b$-axis. The structure can be described as a perovskite with an oxygen deficit. In Fig. 7, $\mathrm{B}$ is the metallic cation $\mathrm{Cu}$ (small radius), surrounded by six oxygen ions occupying site $\mathrm{X}$. The metallic cation site $\mathrm{A}$ is occupied by yttrium (large radius than B). By eliminating oxygen atoms from the ideal perovskite lattice, we obtain $\mathrm{YBa}_{2} \mathrm{Cu}_{3} \mathrm{O}_{7}$ (Fig. 12(a)). Its unit cell contains A layer of $\mathrm{Cu}-\mathrm{O}$ having copper $(\mathrm{Cu} 1)$ surrounded by four oxygen ions, a layer of $\mathrm{BaO}$, a layer of $\mathrm{Cu}-\mathrm{O}$, where $(\mathrm{Cu} 2)$ is surrounded by five oxygen ions forming a polyhedron, a layer of yttrium, short of four oxygen. Thus, the stacking sequence of the $(a b)$ planes is,

$$
\mathrm{Y}-\mathrm{CuO}-\mathrm{BaO}-\mathrm{CuO}_{2}-\mathrm{BaO}-\mathrm{CuO}_{2}-\mathrm{Y}
$$


There are $\mathrm{Cu}-\mathrm{O}$ chains along the $b$-axis. The presence of oxygen atoms in these chains is essential for superconductivity. We know the general formula of the compound is $\mathrm{YBa}_{2} \mathrm{Cu}_{3} \mathrm{O}_{7-\mathrm{x}}$ where $0 \leq x \leq 1$. The lattice parameters vary with the oxygen content ( $x$-value) and for $x$ $\geq 0.6$, the structure is tetragonal. In $\mathrm{YBa}_{2} \mathrm{Cu}_{3} \mathrm{O}_{6}(x=1)$, there are no chain $\left(\mathrm{O}_{4}\right.$ sites $)$ oxygen and $\mathrm{Cu}$ in $b$-axis is in the $1+$ state (Non-superconducting state). Thus, the unit cell parameters vary with the value of $x$ in $\mathrm{YBa}_{2} \mathrm{Cu}_{3} \mathrm{O}_{7-\mathrm{x}}$ (i.e. $a, b$ and $c$ parameters vary with the oxygen-stoichiometry). This has been depicted in Fig. 14(a, b). The phase diagram of YBCO is shown in Fig. 14 (C). The structure is orthorhombic for $x=0$ (Fig. 13). Penetration depth is $120 \mathrm{~nm}$ in the $\mathrm{ab}$ plane, $800 \mathrm{~nm}$ along the $\mathrm{c}$ axis. Bulk properties depend greatly on the manner of synthesis and treatment because of the effect on crystal size, alignment, and density and type of lattice defects.

YBCO is used for making practical superconducting wires. Using YBCO the second-generation HTS wires (referred to as $2 \mathrm{G}$ ) have been manufactured. YBCO can be used to grow High- $T_{c}$ SQUIDS with grain-boundary Josephson junctions. Their higher operating temperature than conventional SQUIDs makes them practical in the study of living biological materials.

\section{(a)}

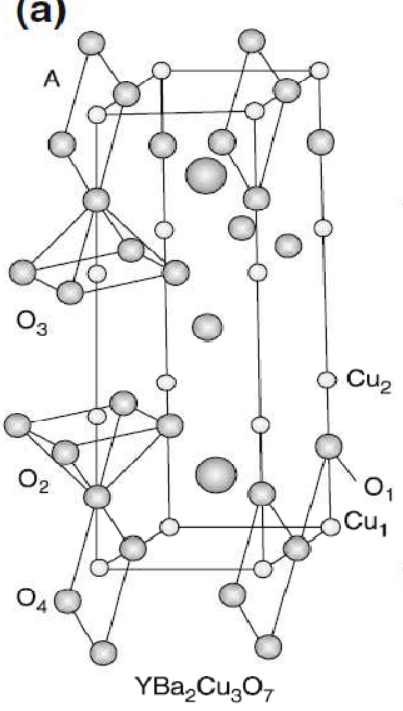

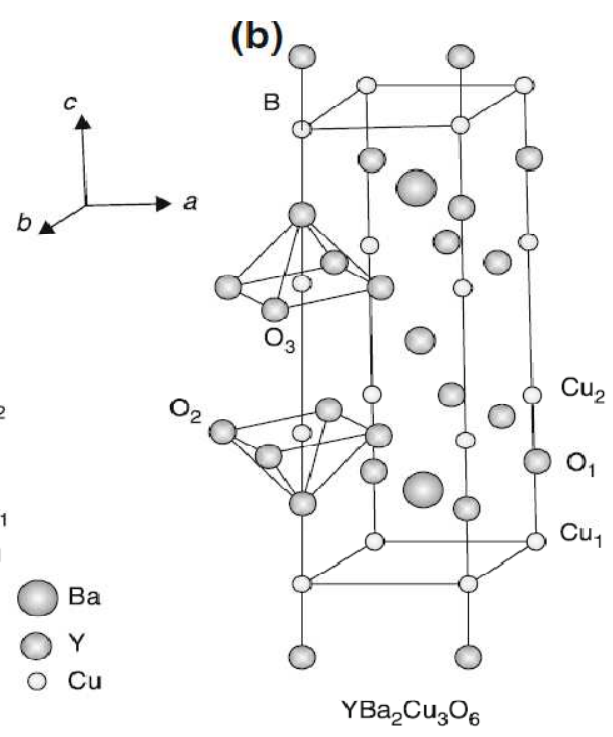

Fig. 12. The structure of $Y B C O(a)$ orthorhombic structure $\left(a_{0}=3.823 \AA, b_{0}=3.885 \AA\right.$, $\left.c_{0}=11.7 \AA\right)$ (b) tetragonal structure $\left(a_{o}=b_{o}=3.86 \AA, c_{o}=11.7 \AA\right)$.
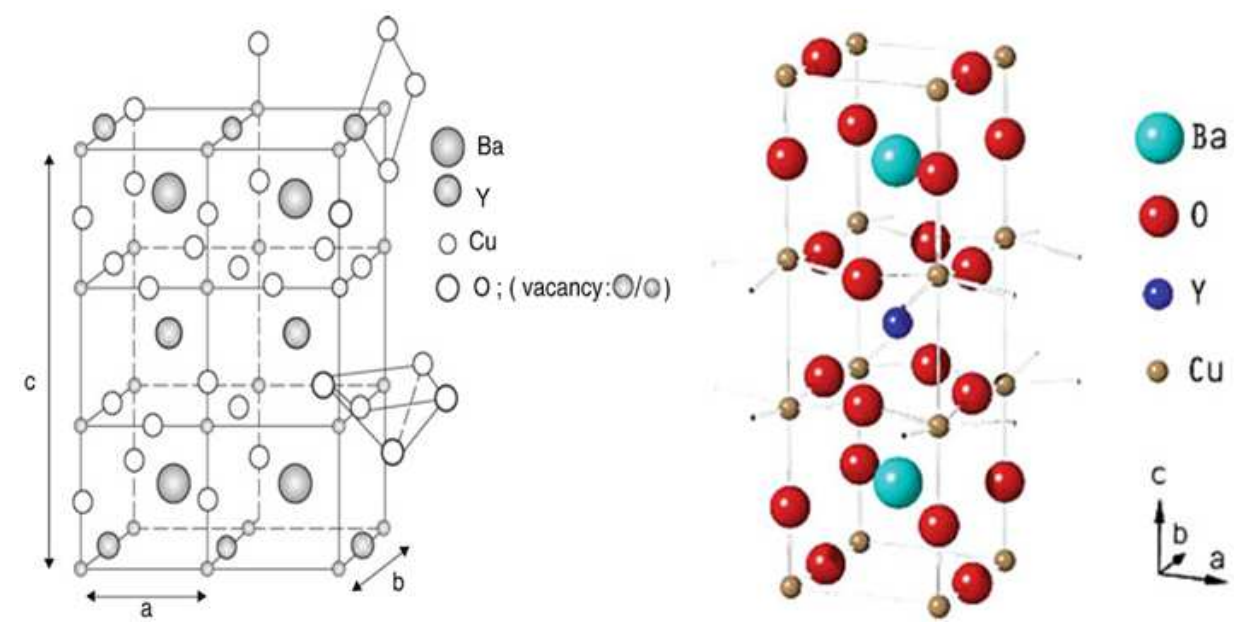

Fig. 13. Crystal structure with oxygen disappearing from alternate chains in $\mathrm{YBa}_{2} \mathrm{Cu}_{3} \mathrm{O}_{6.5}$ and complete disappearance of oxygen from $\mathrm{YBa}_{2} \mathrm{Cu}_{3} \mathrm{O}_{7-x}$ to give $\mathrm{YBa}_{2} \mathrm{Cu}_{3} \mathrm{O}_{6}$. 

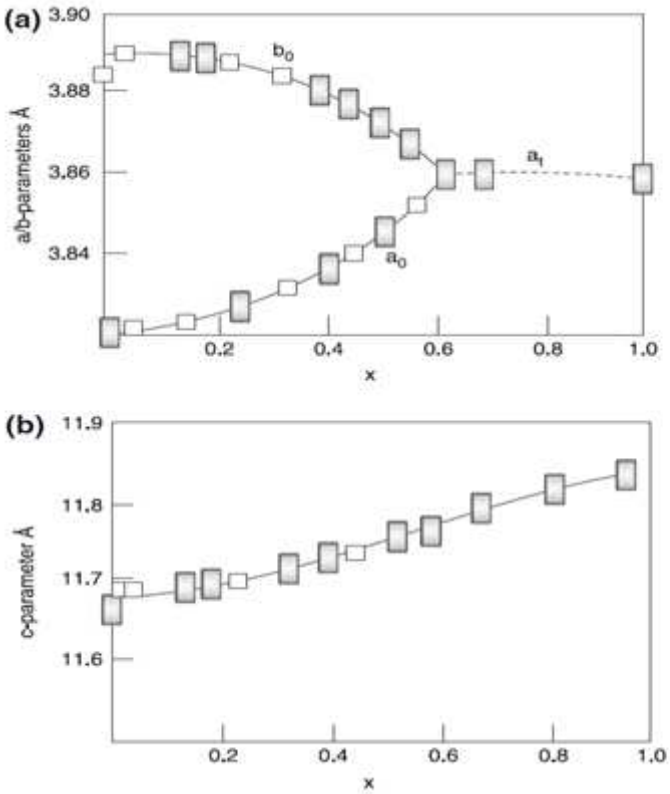

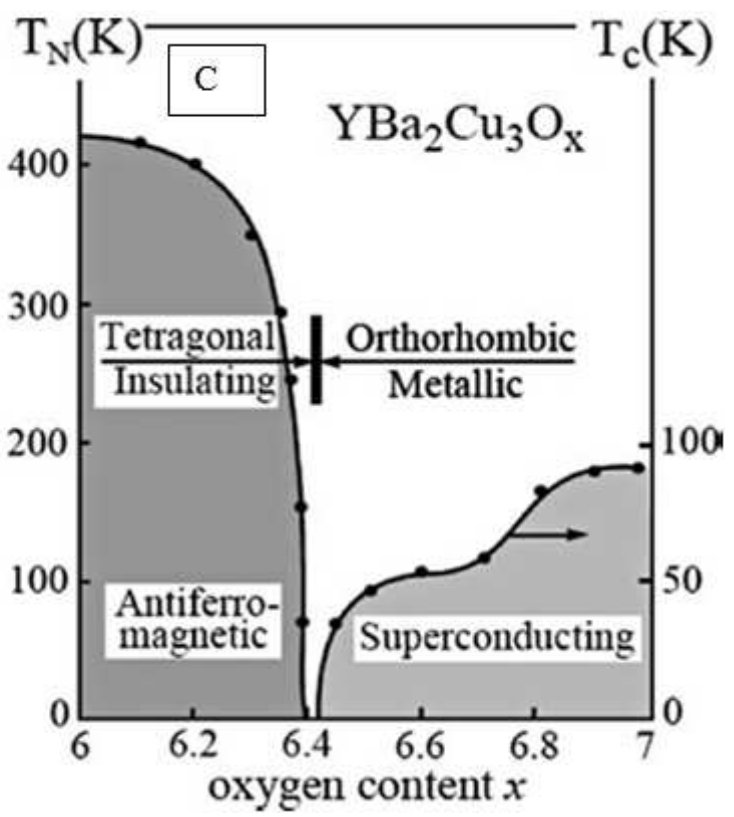

Fig. 14. Variation of the unit cell parameters of $\mathrm{YBa}_{2} \mathrm{Cu}_{3} \mathrm{O}_{7-x}$ with the oxygen stoichiometry $x$. The oxygen deficiency leads to departure from orthorhombicity $(b-a / b+a)$. (C) Phase diagram of $Y B a C u O$.

\subsection{BSCCO Superconductors}

The discovery of the high $T_{c}$ superconductor $\mathrm{La}_{2-\mathrm{x}}(\mathrm{Ba} / \mathrm{Sr})_{\mathrm{x}}$ $\mathrm{CuO}_{4}$ with $T_{c}$ of $40 \mathrm{~K}$ and $\mathrm{YBa}_{2} \mathrm{Cu}_{3} \mathrm{O}_{7-\mathrm{x}}$ with $T_{c}$ of $90 \mathrm{~K}$ led to an intensive search for new oxide superconductors of still higher $T_{c}(>100 \mathrm{~K})$. No new superconductor with $T_{c}$ higher than that of $\mathrm{YBa}_{2} \mathrm{Cu}_{3} \mathrm{O}_{7-\mathrm{x}}$ was reported up to January 1988. In May 1987, Michel et al. ${ }^{45}$ reported the discovery of superconductivity between 7 and $22 \mathrm{~K}$ in rare-earth-less material $\mathrm{Bi}-\mathrm{Sr}-\mathrm{Cu}-\mathrm{O}$. Because of the intense interest in the $90 \mathrm{~K}$ material YBCO, at that time, their report did not attract widespread interest. However, attention quickly focused on the Bi-containing cuprate superconductors in January 1988, when addition of $\mathrm{Ca}$ to the $\mathrm{Bi}-\mathrm{Sr}-\mathrm{Cu}-\mathrm{O}$ ternary led Maeda et al. $^{46}$ to the discovery of bulk superconductivity at $85 \mathrm{~K}$ and evidence of superconductivity up to $110 \mathrm{~K}$ in the $\mathrm{Bi}-\mathrm{Sr}-\mathrm{Ca}-$ $\mathrm{Cu}-\mathrm{O}$ system. The compound with formula $\mathrm{Bi}_{4} \mathrm{Sr}_{3} \mathrm{Ca}_{3} \mathrm{Cu}_{4} \mathrm{O}_{16}$ was found to be responsible for superconductivity at $85 \mathrm{~K}$ in the $\mathrm{Bi}$ system and its structure was established ${ }^{[47,48]}$. The crystal substructure can be viewed as a three dimensional packing of $\mathrm{Bi}_{2} \mathrm{Sr}_{2} \mathrm{CaCu}_{2} \mathrm{O}_{8}$ slabs along the $c$-axis, with the main feature being the presence of the two $\mathrm{Bi}-\mathrm{O}$ layers separated by $3.0 \AA$ and shifted, with respect to each other (crystallographic shear) along the diagonal direction of the perovskite sub-cell. Tarascan et al. ${ }^{49}$ showed the evidence that the $100 \mathrm{~K}$ phase has the $\mathrm{Bi}-2: 2: 2: 3$ formula and its structure contains three $\mathrm{CuO}_{2}$ layers: The three phases of
$\mathrm{Bi}-\mathrm{Sr}-\mathrm{Ca}-\mathrm{Cu}-\mathrm{O}$ can be represented by the general formula $\mathrm{Bi}_{2} \mathrm{Sr}_{2} \mathrm{Ca}_{\mathrm{n}-1} \mathrm{Cu}_{\mathrm{n}} \mathrm{O}_{\mathrm{y}}(n=1,2$ and 3$)$ and have a pseudotetragonal structure (Fig.15a), which can be described as a stacking of a basic $\mathrm{Bi}_{2} \mathrm{Sr}_{2} \mathrm{CuO}_{6}$ unit with either zero, one or two $\mathrm{CaCuO}_{2}$ slabs inserted. The $c$-lattice parameter increases from 24.6 to $30.6 \AA$ and finally to $37.1 \AA$ in going from $n=1$ to 2 and 3 , and the increase results from the progressive addition of $2 \times 1$ and $2 \times 2$ (doubled because of the crystallographic shear) $\mathrm{CaCuO}_{2}$, each about $3 \AA$ thick, to the stacking sequence in the unit cell. For $\mathrm{Bi}_{2} \mathrm{Sr}_{2} \mathrm{CaCu}_{2} \mathrm{O}_{8}$, the stacking sequence is,

$$
\mathrm{Bi}-\mathrm{Sr}-\mathrm{Cu}-\mathrm{Ca}-\mathrm{Cu}-\mathrm{Sr}-\mathrm{Bi} \text {. }
$$

In $\mathrm{Bi}_{2} \mathrm{Sr}_{2} \mathrm{Ca}_{2} \mathrm{Cu}_{3} \mathrm{O}_{10}$, the layer sequence is,

$$
\mathrm{Bi}-\mathrm{Sr}-\mathrm{Cu}-\mathrm{Ca}-\mathrm{Cu}-\mathrm{Ca}-\mathrm{Cu}-\mathrm{Sr}-\mathrm{Bi} \text {. }
$$

The Bi-based high $T_{c}$ superconductors are superior to the YBCO in respect of higher $T_{c}$. This class of superconductors (unlike YBCO) are resistant to water or humid atmosphere and have the advantage of compositional/oxygen stability, e.g. some of its superconducting phases do not gain or lose oxygen, when the material is annealed at $850{ }^{\circ} \mathrm{C}$. Another advantage of the BSCCO materials relates to the fact that $\mathrm{BiO}$ layers being Vander Waal bonded, this material can be easily rolled. This property has been utilized successfully for tape-casting and its texturing. 

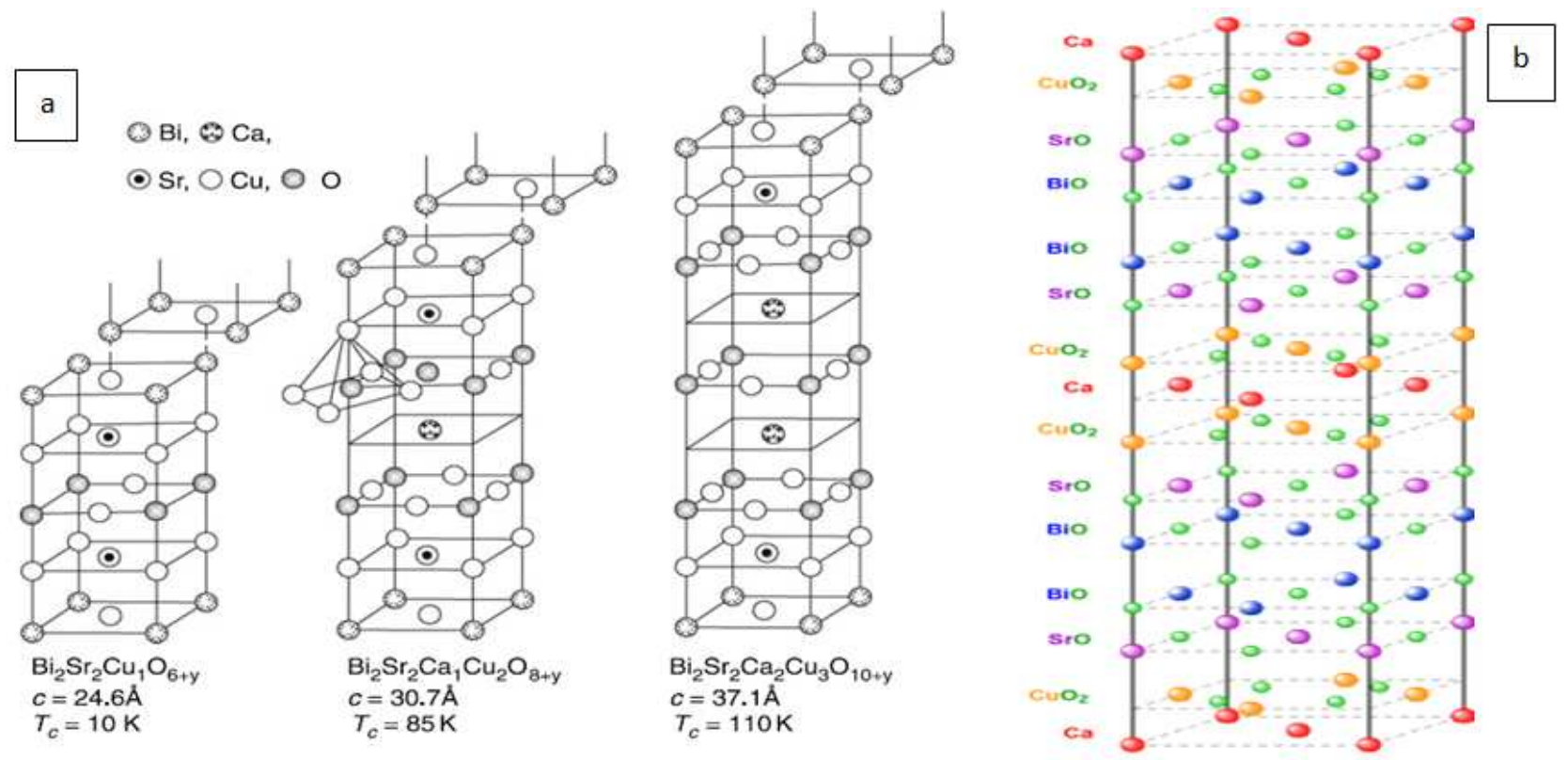

Fig. 15. (a) The crystal structures of the Bi phases of general formula $\mathrm{Bi}_{2} \mathrm{Sr}_{2} \mathrm{Ca}_{n-l} \mathrm{Cu}_{n} \mathrm{O}_{y}$ with $n=1,2$ and 3, (b) The unit cell of high-temperature cuprate superconductor BSCCO-2212.

BSCCO has been more useful so far in bulk applications, has been formed into superconducting wires and placed into the Detroit. Politis ${ }^{50}$ studied multi phase $\mathrm{Sr}-\mathrm{Bi}-\mathrm{Ca}-\mathrm{Cu}-\mathrm{O}$ with the nominal composition of $\mathrm{SrBiCaCu}_{2} \mathrm{O}_{6}$ and observed onset of $T_{c}$ around $120 \mathrm{k}$ and observed double transitions in many samples.

\section{4. ТВССО Superconductors}

Tl-Ba-Ca-Cu-O superconductors have been discovered in 1988 by Sheng and Hermann ${ }^{51-56}$ is the best among all other cuprates due to their high transition temperature, transport critical current densities and low microwave surface resistances. There are two Tl-based superconducting families, TBCCO and TSCCO. TBCCO materials have perovskite like $\mathrm{Ba}_{2} \mathrm{Ca}_{\mathrm{n}-1} \mathrm{Cu}_{\mathrm{n}} \mathrm{O}_{2 \mathrm{n}+1}$ layers and tetragonal crystal structures. The first series of the Tl-based superconductor containing one $\mathrm{Tl}$ $\mathrm{O}$ layer has the general formula $\mathrm{TlBa}_{2} \mathrm{Ca}_{n-1} \mathrm{Cu}_{n} \mathrm{O}_{2 n+3}$, ${ }^{57}$ whereas the second series containing two $\mathrm{Tl}-\mathrm{O}$ layers has a formula of $\mathrm{Tl}_{2} \mathrm{Ba}_{2} \mathrm{Ca}_{n-1} \mathrm{Cu}_{n} \mathrm{O}_{2 n+4}$ with $n=1,2$ and 3 . In the structure of $\mathrm{Tl}_{2} \mathrm{Ba}_{2} \mathrm{CuO}_{6}(\mathrm{Tl}-2201)$, there is one $\mathrm{CuO}_{2}$ layer with the stacking sequence,

$$
(\mathrm{Tl}-\mathrm{O})(\mathrm{Tl}-\mathrm{O})(\mathrm{Ba}-\mathrm{O})(\mathrm{Cu}-\mathrm{O})(\mathrm{Ba}-\mathrm{O})(\mathrm{Tl}-\mathrm{O})(\mathrm{Tl}-\mathrm{O}) \text {. }
$$

In $\mathrm{Tl}_{2} \mathrm{Ba}_{2} \mathrm{CaCu}_{2} \mathrm{O}_{8}$ (T1-2212), there are two $\mathrm{Cu}-\mathrm{O}$ layers with a $\mathrm{Ca}$ layer in between. Similar to the $\mathrm{Tl}_{2} \mathrm{Ba}_{2} \mathrm{CuO}_{6}$ structure, $\mathrm{Tl}-\mathrm{O}$ layers are present outside the $\mathrm{Ba}-\mathrm{O}$ layers. In $\mathrm{Tl}_{2} \mathrm{Ba}_{2} \mathrm{Ca}_{2} \mathrm{Cu}_{3} \mathrm{O}_{10}$ (T12223), there are three $\mathrm{CuO}_{2}$ layers enclosing $\mathrm{Ca}$ layers between each of these. In Tl-based superconductors, $T_{c}$ is found to increase with the increase in $\mathrm{CuO}_{2}$ layers. However, the value of $T_{c}$ decreases after four $\mathrm{CuO}_{2}$ layers in $\mathrm{TlBa}_{2} \mathrm{Ca}_{n-1} \mathrm{Cu}_{n} \mathrm{O}_{2 n+3}$, and in the $\mathrm{Tl}_{2} \mathrm{Ba}_{2} \mathrm{Ca}_{n-}$ ${ }_{1} \mathrm{Cu}_{n} \mathrm{O}_{2 n+4}$ compounds, it decreases after three $\mathrm{CuO}_{2}$ layers. ${ }^{58}$ The structure of T1-2201, T1-2223 and T1-2212 are shown in Fig. 16.

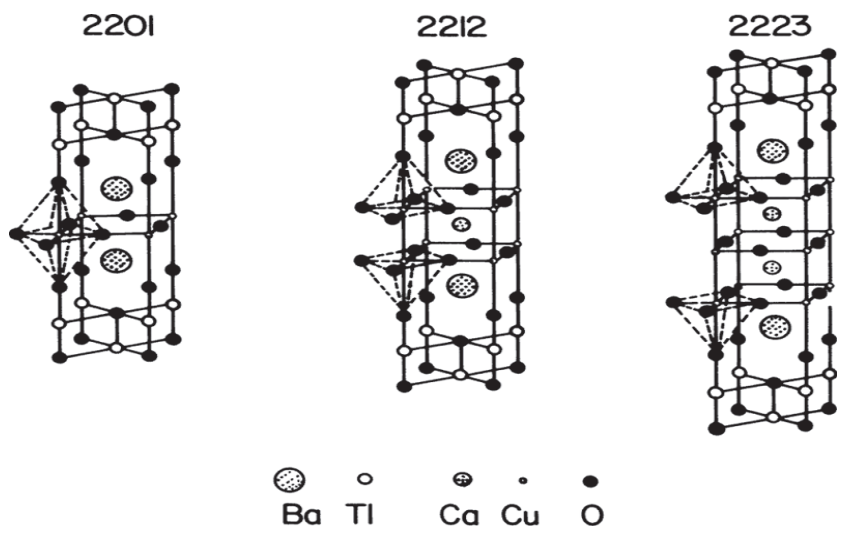

Fig. 16. Crystal structure of the layered $\mathrm{Tl}-\mathrm{Ba}-\mathrm{Ca}-\mathrm{Cu}$-O compounds.

\section{5. $\mathrm{Hg}-\mathrm{Ba}-\mathrm{Ca}-\mathrm{Cu}-\mathrm{O}$ Superconductors}

Mercury bearing compound $\mathrm{HgBa}_{2} \mathrm{RCu}_{2} \mathrm{O}_{6+\mathrm{x}}$ ( $\mathrm{Hg}$ : 1212), where $\mathrm{R}$ is a rare-earth element, was synthesised by Putilin et al. $^{59}$. It has a structure similar to the thallium bearing superconductor $\mathrm{TlBa}_{2} \mathrm{CaCu}_{2} \mathrm{O}_{7}$ (Tl-1212), which has one $\mathrm{TlO}$ layer and two $\mathrm{CuO}_{2}$ layers per unit cell and $T_{c}$ is $85 \mathrm{~K}^{60}$. But inspite of the resemblance to $\mathrm{Tl}: 1212, \mathrm{Hg}: 1212$ was found to be non-superconducting. In 1993, Putilin et al. ${ }^{61}$ reported the synthesis of the compound $\mathrm{HgBa}_{2} \mathrm{CuO}_{4+\mathrm{x}}(\mathrm{Hg}: 1201)$ with only one $\mathrm{CuO}_{2}$ layer per unit cell. It was found to be superconducting below $94 \mathrm{~K}$. Its structure is similar to that of Tl: $1201\left(T_{c} \leq 10 \mathrm{~K}\right)$, but its $T_{c}$ is considerably higher. The availability of such a material with high $T_{c}$ and a single metal-oxide $(\mathrm{HgO})$ layer may be important for technological applications, because a smaller spacing between $\mathrm{CuO}_{2}$ planes leads to better superconducting properties in a magnetic field. The structural arrangement of $\mathrm{HgBa}_{2} \mathrm{CuO}_{4+\mathrm{x}}$ is similar to that of $\mathrm{TlBa}_{2} \mathrm{CuO}_{5}$, except for the oxygen stoichiometry of the 
$\mathrm{HgO}_{\mathrm{x}}$ and $\mathrm{TlO}_{1-\mathrm{x}}$ layers, respectively. For the former, $\mathrm{x}$ is very small and for the latter, the $\mathrm{TlO}_{1-\mathrm{x}}$ layer is only slightly oxygen depleted. These different requirements for attaining the optimal concentration of holes (carriers) are due to the different preferred coordination geometries of the $\mathrm{Tl}^{3+}$ and $\mathrm{Hg}^{2+}$ cations. The rare-earth based $\mathrm{Hg}$ : 1212 compounds were not found to be superconducting because the hole concentration in these phases might not be high enough for inducing superconductivity. Putilin et al. ${ }^{62}$ replaced the trivalent rare-earth cation by divalent $\mathrm{Ca}^{2+}$ and obtained a superconductive transition temperature of above $120 \mathrm{~K}$ in $\mathrm{HgBa}_{2} \mathrm{CaCu}_{2} \mathrm{O}_{6+\mathrm{x}}$. In 1993, Schilling et al. ${ }^{63}$ reported their discovery of superconductivity above $130 \mathrm{~K}$ in a material containing $\mathrm{HgBa}_{2} \mathrm{Ca}_{2} \mathrm{Cu}_{3} \mathrm{O}_{8+\mathrm{x}}$ (with three $\mathrm{CuO}_{2}$ layers per unit cell) and $\mathrm{HgBa}_{2} \mathrm{CaCu}_{3} \mathrm{O}_{6+\mathrm{x}}$ (with two $\mathrm{CuO}_{2}$ layers per unit cell) and an ordered superstructure comprising a defined sequence of the unit cells of these phases (Fig. 17). A maximum transition temperature of $133 \mathrm{~K}$ had been obtained which was distinctly higher than the previously established value of $125-127 \mathrm{~K}$ observed in $\mathrm{Tl}_{2} \mathrm{Ba}_{2} \mathrm{Ca}_{2} \mathrm{Cu}_{3} \mathrm{O}_{10}$. In September 1993, a record $T_{c}$ of $150 \mathrm{~K}$ in $\mathrm{HgBa}_{2} \mathrm{Ca}_{2} \mathrm{Cu}_{3} \mathrm{O}_{8+\mathrm{x}}$ at quasihydrostatic pressure of $150 \mathrm{~K}$-bar was obtained by $\mathrm{Chu}$ (Texas Centre for Superconductivity, U.S.A.).

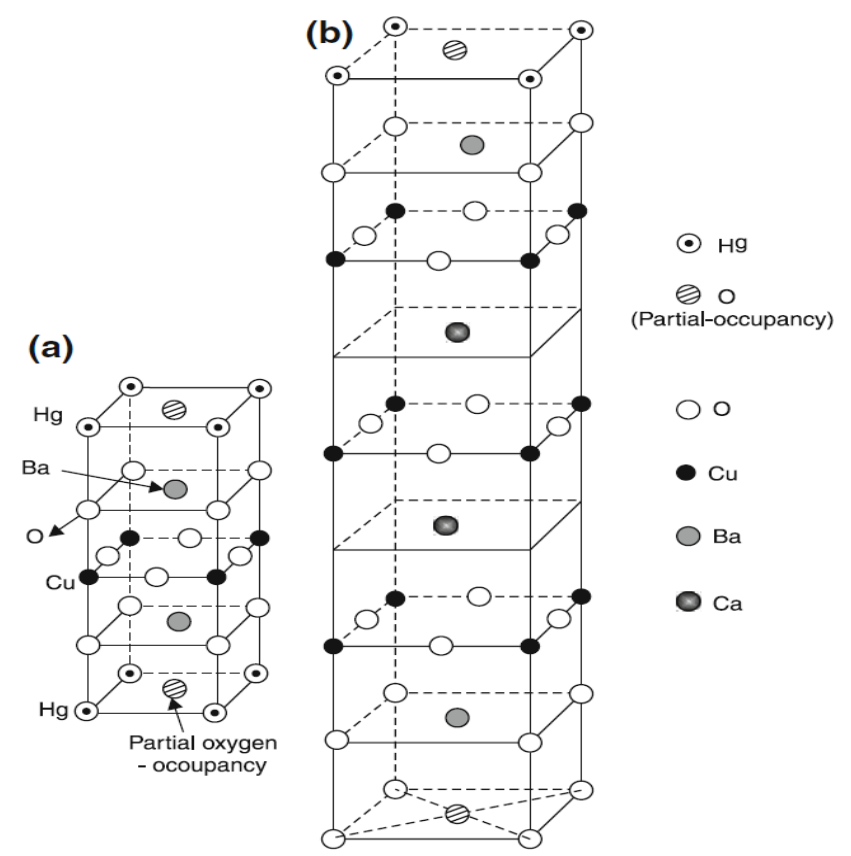

Fig. 17. Atom cluster model for central cell of (a) Hg-1201 and (b) Hg-1223 superconductors.

Such pressure effect on $T_{c}$ might also be duplicated by chemical means, i.e. "chemical pressure" imposed by other "element" substituted on $\mathrm{Hg}$-site (published in Nature) (Previous record of $T_{c}$ of $135 \mathrm{~K}$ in $\mathrm{Hg}$ : 1223 was at ambient pressure). Table 2 gives lattice parameters of $\mathrm{Hg}$ superconductors.
Table 2. Lattice parameters of Hg based superconductors.

\begin{tabular}{lll}
\hline Cuprate & $\boldsymbol{T}_{\mathbf{c}}{ }^{\mathbf{a}}(\mathbf{k})$ & Lattice Parameters \\
\hline $\mathrm{Hg}-1201$ & 94 & $\mathrm{a}=\mathrm{b}=3.85 \AA, \mathrm{c}=9.5 \AA$ \\
$\mathrm{Hg}-1212$ & 120 & $\mathrm{a}=\mathrm{b}=3,85 \AA, \mathrm{c}=12.6 \AA$ \\
$\mathrm{Hg}-1223$ & 133 & $\mathrm{a}=\mathrm{b}=3.85 \AA, \mathrm{c}=15.7 \AA$ \\
\hline
\end{tabular}

\section{Application of Cuprate Based Superconductors}

Due to the unique properties of high temperature superconducting materials, their applications are becoming attractive continuously with the improvement in properties of superconductors. The search for applications has always been a driving force for superconductor materials science. Right from the discovery, it had been envisioned that superconducting (SC) coils with high persistent current might be used to produce strong magnetic field.

In the last ten years, many applications of hightemperature superconductors have been developed. In this section some typical applications of cuprate based superconductors will be discussed.

\subsection{Superconducting Bulk}

Superconducting bulk YBCO is grown using half-metal materials at a high temperature of nearly $1000^{\circ} \mathrm{C}$, followed by a very slow cooling. The special feature of the bulk is that the introduction of pinning centers is easily carried out by controlling the density of fine $\mathrm{Y}_{2} \mathrm{BaCuO}_{5}$ particles as pinning centers in the bulk. The most distinguishing characteristic is that it is possible to trap a strong magnetic field of 2 to $3 \mathrm{~T}$, even at liquid nitrogen temperature. This value is much higher than the magnetic field of an ordinary permanent magnet. Superconducting bulk has been applied as a bearing system that is used to store electricity in a flywheel system, as shown in Fig. 18. The capacity of this system is about 10 $\mathrm{KW} / \mathrm{h}$ and it has operated safely for many months. The second application is a magnetic separation system for the water cleaning made by Hitachi, Ltd. The impurity particles in water join magnetic particles, and they are removed from the filter by the strong magnetic field of the bulk. The operation can be continuous and results have been impressive.

\subsection{Superconducting Magnet for Maglev Trains}

By using Bi-2223 superconducting tape magnet for maglev train has been made. This magnet has a racetrack shape and consists of twelve pancake coils, of $1 \mathrm{~m}$ length and $50 \mathrm{~cm}$ height, as shown in Fig.19. This magnet is operated at 20k and generates a magnetic field of $2.5 \mathrm{~T}$ at the center of the magnet. Furthermore, the rate of decay of the persistent current is only $0.5 \%$ a day. This magnet is very successful and recently a train using this magnet reached a speed of 500 $\mathrm{km} / \mathrm{h}$. 

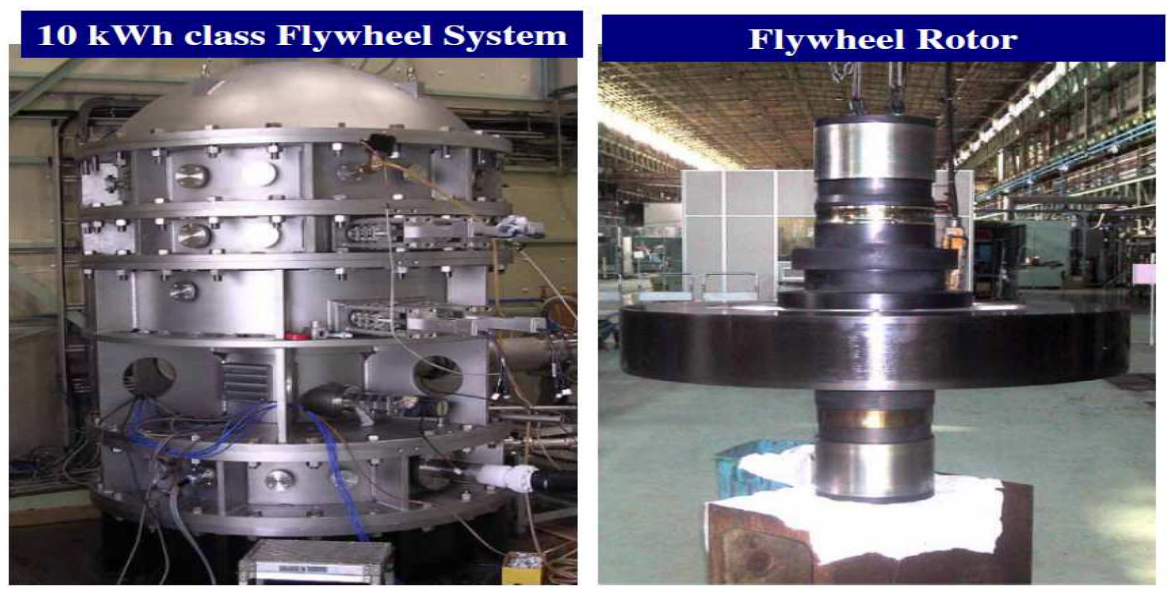

Fig. 18. Electricity storage system of flywheel type.

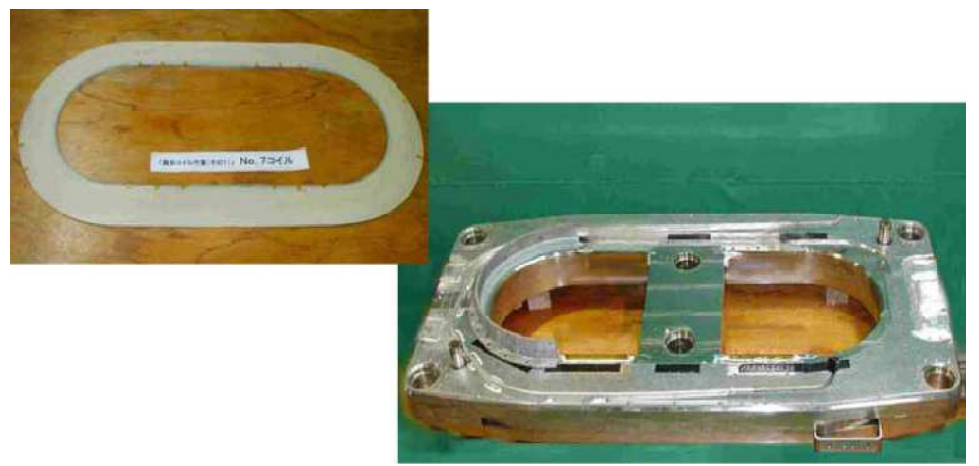

Fig. 19. Development of racetrack-shaped coil manufacturing technology.

\subsection{Superconducting Motor for Ships}

The ship propulsion system is undergoing a revolution. In the new system, propellers and the electric motor are directly connected and they are outside the body of the ship, as shown in Fig. 20. By employing such a system, freedom of boat design is very much improved, and as a result, energy saving becomes possible. This propulsion system is called a "pod motor", and a superconducting motor is most suitable for this pod motor, because the superconducting motor generates a large torque even at a slow rotating propeller speed of about $100 \mathrm{rpm}$; furthermore, it is much smaller and much lighter than ordinary motors using copper wire. In the United States, various types of superconducting motors are being developed; all of them use Bi-2223 superconducting tape. In Japan, a superconducting motor using YBCO tape has been recently developed, and this is the first such motor in the world. ${ }^{33}$

(a)

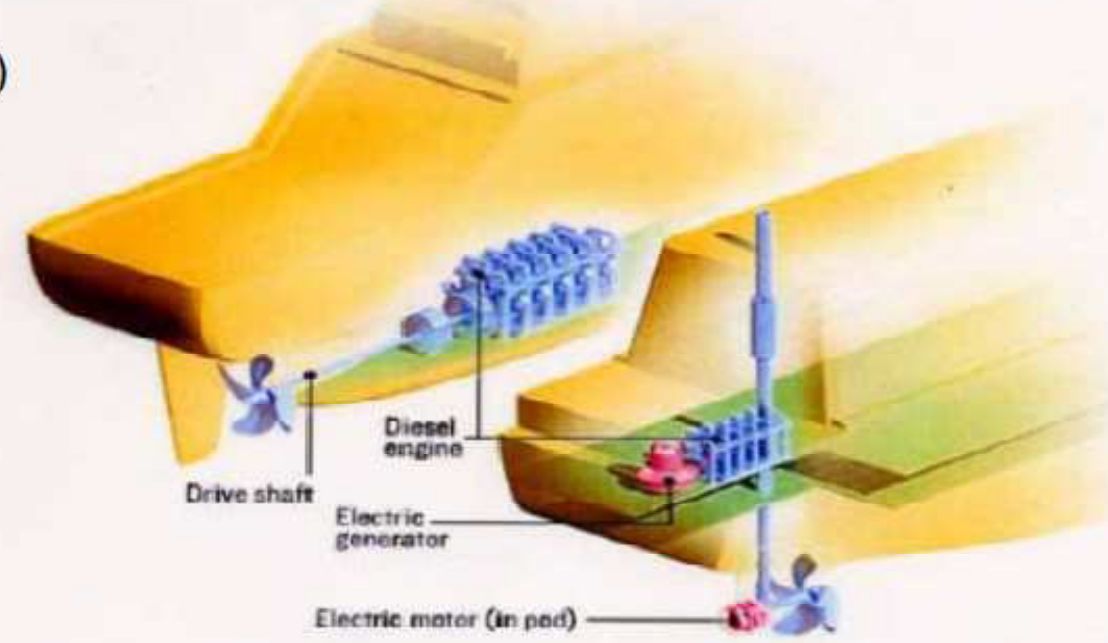

Fig. 20. Comparison of propelling system; (a) ordinary system and (b) new system with pod motor. 


\subsection{SQUID}

The structure and characteristics of the SQUID system using a low-temperature $\mathrm{Nb}$ superconductor are well known. It shows very high sensitivity to a magnetic field. In the case of using high temperature superconductors such as YBCO, the superconducting thin film is sputtered on the plate of a bicrystal, and Josephson junctions are formed on the crystal boundary. The sensitivity of SQUID systems using YBCO operating at $77 \mathrm{k}$ is about ten times lower than that of an $\mathrm{Nb}$ based system operating at $4 \mathrm{~K}$, but by improving the manufacturing process and the design, the sensitivity will be increased in the near future. Note that a SQUID system using an Hg-based high-temperature superconductor showed low flux noise level several times smaller than that using YBCO at $77 \mathrm{~K}$. The application of high temperature SQUIDs to a magneto cardiograph has been made recently by Hitachi, Ltd. ${ }^{33}$ It consists of 51 SQUIDs on one plate and is used for the diagnosis of human heart diseases. ${ }^{33}$

\subsection{Transmission of Commercial Power}

An idealized application for superconductors is to use them in the transmission of commercial power to cities. However, due to the high cost and needlessness of cooling miles of superconducting wire to cryogenic temperatures, this has only happened with short "test runs." In May of 2001 some 150,000 residents of Copenhagen, Denmark, began receiving their electricity through high-temperature Superconducting material. That cable was only $30 \mathrm{~m}$ long, but proved sufficient for testing purposes. This marked the first time commercial power has been delivered to customers through superconducting wire. Superconducting transformers with HTSC windings have been carried out in Japan and the USA. Super-Foam, synthesized from $\mathrm{YBa}_{2} \mathrm{Cu}_{3} \mathrm{O}_{x}$ may be ideal material for the fault current limiters.

\subsection{Bio-Magnetism}

An area where superconductors can carry out a life-saving function is in the field of bio-magnetism. Doctors need a non-invasive means of determining what's going on inside the human body. By impinging a strong superconductor derived magnetic field into the body, hydrogen atoms that exist in the body's water and fat molecules are forced to accept energy from the magnetic field. They then release this energy at a frequency that can be detected and displayed graphically by a computer. Magnetic Resonance Imaging (MRI) was actually discovered on July 3, 1977. It took almost five hours to construct one image.

\subsection{Electric Power Applications}

\subsubsection{Superconducting Tapes and Cables}

A possibility of electric energy transportation without loss is the most attractive property of superconductors. A high transition temperature of the cuprate HTS has enabled their practical applications by development of HTS power cables, which are able to carry up to ten times greater current and power capacity per cross section than conventional copper cables (see, e.g., $[66,67])$. In the first generation $(1 \mathrm{G})$ of the HTS wires, the Bi-2212 material was used in the "powder in tube" method: a silver tube is filled under high pressure with Bi-2212 powder. Due to a mica- or graphite-like structure, the Bi-2212 material forms long filaments of the order of 10 $\mu \mathrm{m}$ thick and $200 \mu \mathrm{m}$ long, which is being inserted in a silver tube produce a wire with good mechanical properties. The Bi-based wires were discovered in Japan and presently long cables up to $1,500 \mathrm{~m}$ are produced by Sumitomo Electric Company (http://www.sumitomo.com). The HTS cables have significantly lower impedance than conventional cables, which is important for efficient transportation of AC electric power within the grid. Typically, $1 \mathrm{G}$ wires have self field critical currents $\sim 180 \mathrm{~A}$ at $77 \mathrm{~K}\left(J_{\mathrm{ct}} \sim 10^{4} \mathrm{Acm}^{-2}\right)$, which has enabled to use them in various applications.

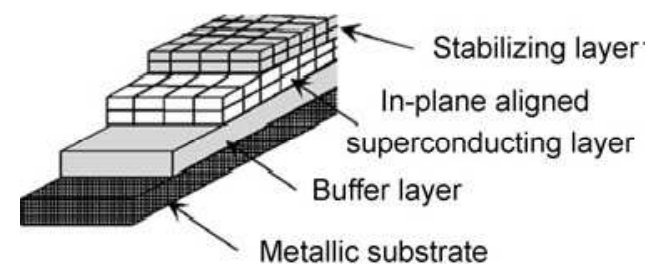

Fig. 21. Schematic geometry for the $2 G$ coated-conductor.

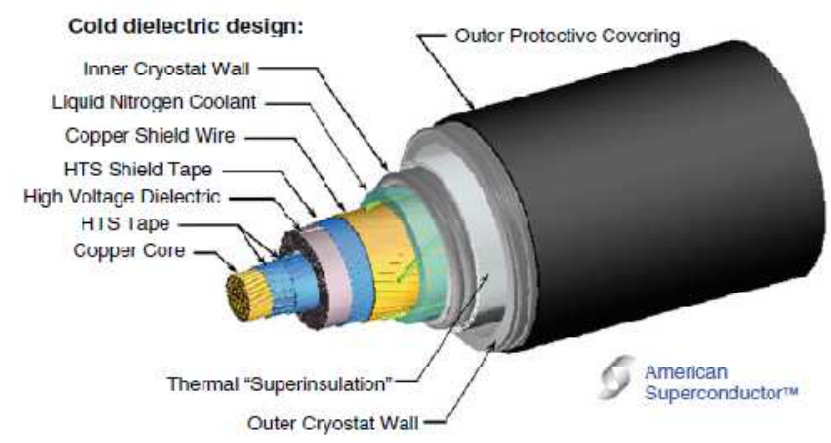

Fig. 22. Single-phase HTS cable demonstrated to $138 \mathrm{kV}$.

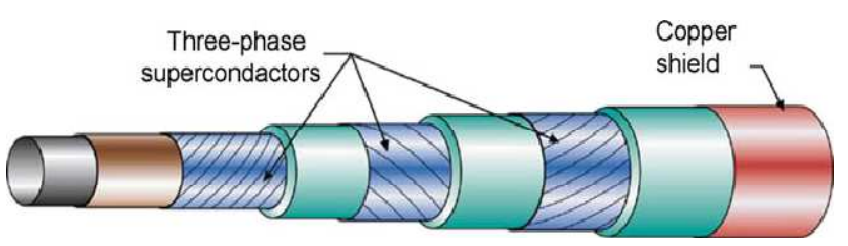

Fig. 23. Three-phase low impedance HTS cable "Triax".

However, the $1 \mathrm{G}$ wire has several disadvantages: their critical current density $J \mathrm{c}$ strongly reduced under magnetic field and their production demands a large amount of expensive silver presenting an obstacle to their wide commercial application. Therefore, a development of a new second generation $(2 \mathrm{G})$ of wires fabricated on the basis of YBCO compounds has accelerated superconducting cable commercial applications. The $2 \mathrm{G}$ wires are manufactured in the form of the tapes by deposition of about $1 \mu \mathrm{m}$ thick YBCO epitaxially grown layers on the textured nickel-alloy 
substrate with several buffer layers in between as shown in Fig. 21. The tape is coated by the stabilizing layers made of copper, brass, or stainless steel (for details, see [68]). The YBCO $2 \mathrm{G}$ tapes exhibit critical current densities of the order of $250 \mathrm{~A}$ per $\mathrm{cm}$ width or $J_{\mathrm{ct}} \sim 3 \times 10^{6} \mathrm{Acm}^{-2}$ (77K, self field), much higher than $1 \mathrm{G} \mathrm{Bi}$-based wires. Two processes used in the manufacture of the tapes: ion beam assisted deposition (IBAD) and rolling assisted biaxially textured substrates (RABiTS) can be carried out in a reel-to-reel configuration, which greatly accelerates the production. Critical current densities up to $\sim 700 \mathrm{~A}$ per $\mathrm{cm}$ width at $77 \mathrm{~K}$ were reported by SuperPower Inc., in short, $\sim 1 \mathrm{~m}$ tapes produced by real-toreal continuous process [69]. The critical current properties in the Bi-2223 silver-sheathed tapes and the YBCO coated conductors and a possibility of further improvement of their properties is discussed, for example, by Matsushita et al. [70]. These HTS coated conductor tapes are used in fabricating power cables. An example of the single-phase power cable produced by American Superconductor Company (AMSC) (http://www.amsc.com) is shown in Fig. 22. A higher than in YBCO tapes critical current has been obtained in the GdBCO coated HTS conductor. By using a pulsed laser deposit technique for fabricating the GdBCO tapes, critical current densities $J_{c t} \sim 300 \mathrm{~A}$ per $\mathrm{cm}$ width was achieved at $77 \mathrm{~K}$ (self field) and $J_{\mathrm{ct}} \sim 40 \mathrm{~A}$ per $\mathrm{cm}$ width under the parallel magnetic field 3T. To reduce HTS cable costs, three-phase power cables were developed. Figure 23 shows the three-phase low impedance HTS $13.4 \mathrm{kV}$ cable developed by AMSC, where three $2 \mathrm{G}$ wires are concentrically placed around the common central core, surrounded by a copper shield. The Triax HTS cable was developed jointly by Southwire and nkt cables companies. This compact construction has several advantages over the single-phase cable: in the three-phase cable, only about one-half of the quantity of HTS wire is needed and the cables cold surface area is reduced, which lower the cost associated with cryogenic cooling equipment. A single HTS Triax cable operating at $13 \mathrm{kV}$ carries $3,000 \mathrm{~A}$, which is equivalent of 18 conventional underground cables.

\subsubsection{Fault Current Limiters}

To protect the transmission or distribution electric systems from outages caused by fault currents a special device - a fault current limiter (FCL) is installed in the transmission grid. A fault current occurs in the event of a short circuit caused by lightning, accidental contact between the lines or the ground, etc. In this case, the power current flowing through a local network can increase enormously damaging electrical equipment. Conventional line reactors widely used as FCLs have high AC losses and can produce voltage drop in the grid in the case of a fault current. The HTS technology offers a much better solution to the fault current problem and represents one of the most successful applications of the cuprate superconductors. In the HTS FCL, a basic property of a superconductor is used, which is a transition from the zero resistance superconducting state to the normal resistive state when the electric current exceeds the material's critical current. The HTS FCL represents a coated conductor consisted of layers of HTS material within layers of resistive materials. Under normal operating conditions, the current in the cable flows through the HTS layers in the FCL. In the case of a fault, the current exceeds the HTS material's critical current and the HTS layers become normal. In that case, the current is automatically shunted within a millisecond to flow through the higher resistance layers, effectively quenching the fault current amplitude. The very rapidly operated HTS FCLs greatly reduce damage to electrical equipment caused by system faults. They are fail-safe since they require no external sensing of the current to initiate the transition. Several types of FCLs were developed: a single-phase stand alone FCLs of high power or HTS rods and cylinders connected in parallel with conventional copper cables to reduce heating of the FCL and decreasing the recovery time $[66,68]$.

\section{8. ctive Microwave Devices}

The cuprate based superconducting material can be used to make many kinds of active microwave devices. It can be used to make an Infrared detection device. A direct detection of infrared (IR) radiation is based on the breaking of the Cooper pairs by absorption of IR photons. The pair breaking then can be measured from changes of certain superconducting characteristics. More efficient for detection frequencies above $1 \mathrm{THz}$ are the hot-electron bolometers (HEBs), which require100 time's smaller local oscillator power level to drive the device in comparison with the Schottky-diode mixer. In the IR detection by a HEB, a nonlinearity of the resistivity close to the superconducting phase transition is employed. It is convenient to use the HEB at temperatures much lower than the transition temperature but to operate the device below but very close to the critical current by applying an external voltage. Absorption of electromagnetic waves generates "hot" electrons resulting in heating of the HEB, which changes its $I-V$ characteristic. The HEBs using YBCO films could be attractive since they can work at temperatures accessible with low-power cryocoolers.

\subsection{Single-Quantum Flux (SFQ) Devices}

In the past ten years, the circuit technology of SFQ circuits using Nb-based SFQs has made a good progress, the integration of more than $10^{4}$ junctions has become possible, and small-scale MPU, high-speed shift registers and highspeed switching systems have been developed. Special feature of SFQ circuits based on high-temperature superconductors are a high-speed operation of over $100 \mathrm{GHz}$ and a high-temperature operation at approximately $40 \mathrm{~K}$. On the other hand, the integration is more difficult than that of $\mathrm{Nb}$-based SFQ circuits owing to the complexity of process technology. In this report, two types of SFQ circuits are shown, one is a high-speed sampler and the other is a toggle flip-flop circuit. In both of them, about twenty Josephson junctions are used and operated at about $40 \mathrm{~K}$. 

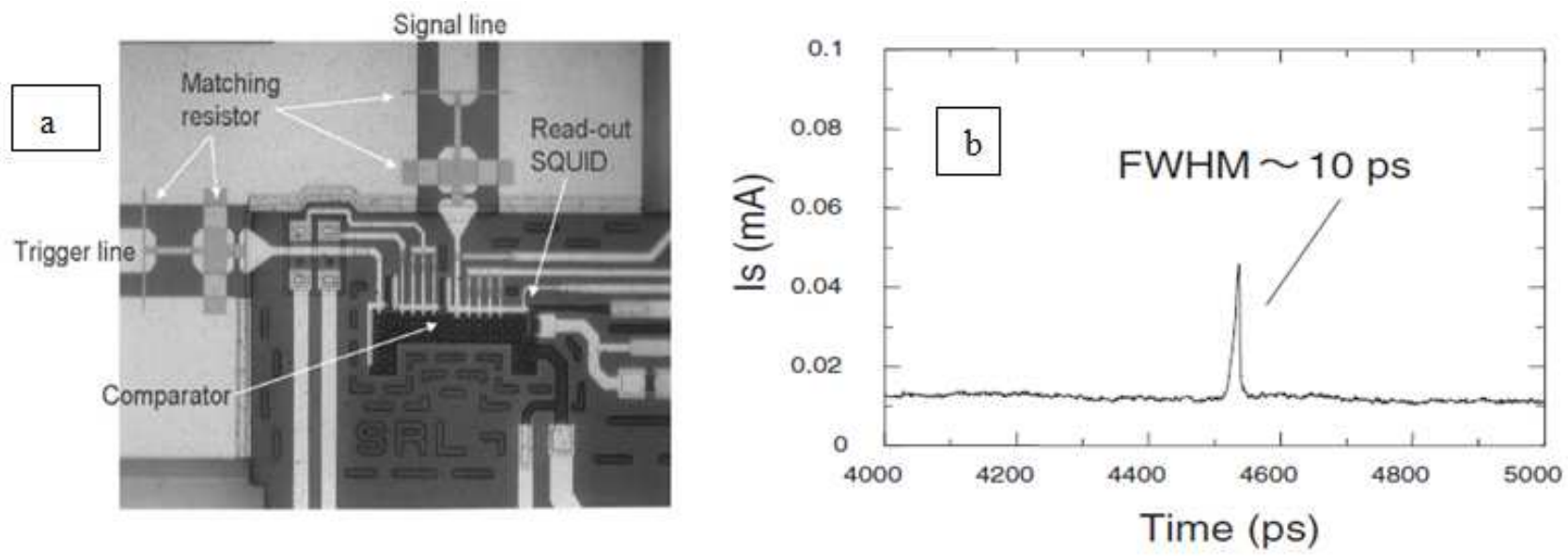

Fig. 24. (a) Photomicrograph of fabricated sampler chip including matching circuits. (b) Measured waveform of short-duration pulse generator.
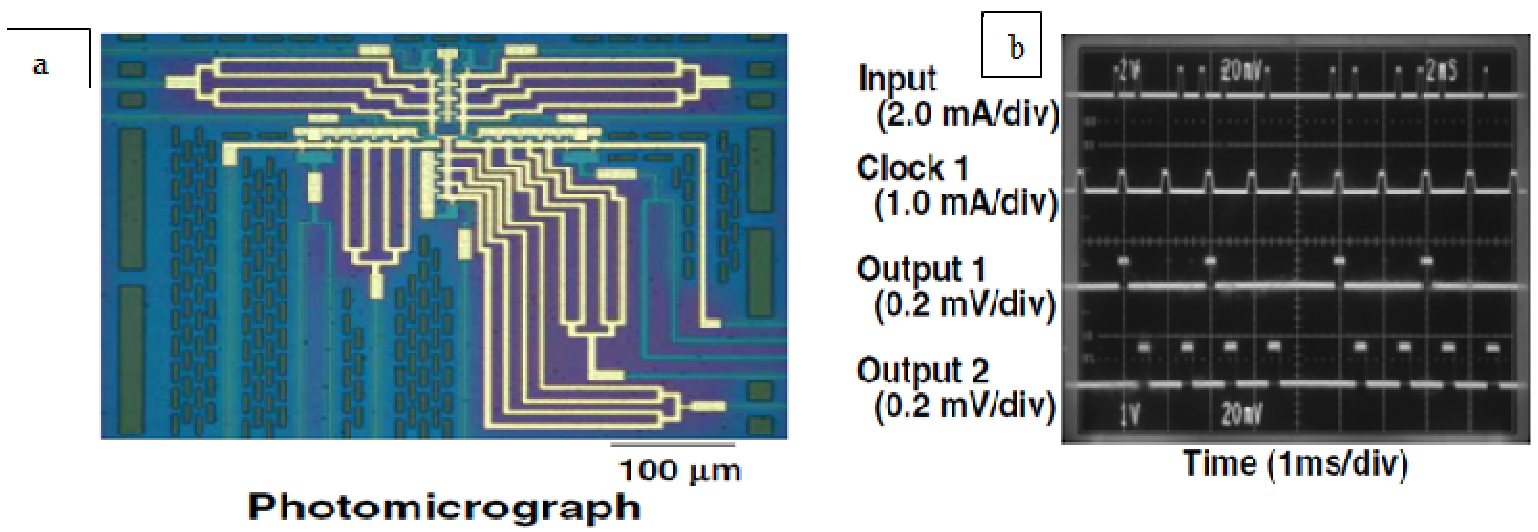

Fig. 25. Fabricated 1:2 switch circuit.

The high-speed sampler is very useful for observing waveforms of a very short pulse greater than $40 \mathrm{GHz}$, which will be popular in future, communication systems. Figure 24 is a photograph of the circuit of the sampler using YBCO junctions, and the obtained waveform of a high speed pulse. ${ }^{33}$ The toggle flip-flop circuit generates two output pulses for one input, and can be used as demultiplexer. Figure 25 is a photograph of such a circuit, and the waveforms of one input and two output signals are shown for comparison. This circuit is operated faster than $370 \mathrm{GHz} .{ }^{33}$ At present, the integration of a high-temperature SFQ circuit is limited to about 100 junctions, but it is hoped that this will reach to more than 500 junctions in future.

\subsection{Scientific Research}

Magnets for plasma confinement and superconducting cables are two extreme examples of applications for which a higher upper critical field at low temperature and a high critical temperature are respectively the desired characteristics to be achieved. Higher fields are desirable to improve plasma confinement, and probably cannot be achieved with low temperature superconductors. Superconducting magnets have potential application for highenergy physics research. An accelerator of high energetic charged particles (Large Hadron Collider) is constructed in
Europe by CERN consortium in 2007. The parameters of HTSC origin are sufficient to produce multi-charged ions.

\section{Conclusion}

The discovery of high temperature superconductivity is one of the most brilliant events in the $20^{\text {th }}$ century, and it led to the wider physical concept of a "strongly correlated electron system". This system is extremely complicated; because, the physics of high-temperature superconductivity is not thoroughly understood yet. Till this moment hightemperature superconducting properties has been discovered within many types of materials. In this review paper we have tried to discuss about cuprate based superconducting materials. We have studied the crystal structures, phase diagram, characteristics and different properties of cuprate based superconductors. Also we have studied the applications of cuprate based superconductors in different sectors. As the high temperature superconductors are brittle the future applications of HTS is very exciting. It is necessary to overcome this problem. However many researchers have tried to solve this problem and we think near future they will be succeed. We hope that the topics described in this overview will be helpful for the discovery of new practical and possible applications of cuprate-based high temperature superconductors. 


\section{References}

[1] W. Buckel, R. Kleiner, Supraleitung - Grundlagen and Anwendungen, WILEY - VCH Verlag, Weinheim (2013); W. Buckel, R. Kleiner, Superconductivity - Fundamentals and Applications, WILEY-VCH Verlag, Weinheim (2004).

[2] R. A. Shukor, High Temperature Superconductors: Materials, Mechanisms and Applications, Akademi Sains, Malaysia (2009).

[3] A. Vilenkin and E. P. S. Shellard, Cosmic Strings and other Topological Defects, Cambridge University Press, Cambridge (1994).

[4] A. Sedrakian, Phys. Rev. D 71, 083003 (2005).

[5] M. G. Alford, A. Schmitt, K. Rajagopal, and T. Schafer, Rev. Mod. Phys. 80, 1455 (2008).

[6] T. H. Geballe, Science 293 (2001) 223.

[7] E. A. Ekimov, V. A. Sidorov, E. D. Bauer, N. N. Mel'nik, N. J. Curro, J. D. Thompson, S. M. Stishov, Nature 428 (2004) 542.

[8] C. Buzea, T. Yamashita, Supercond. Sci. Techn. 14 (2001) R115.

[9] J. Bardeen, L. N. Cooper, and J. R. Schrieffer: Phys. Rev. 108 (1957) 1175.

[10] J. R. Schrieffer: Theory of Superconductivity (Westview Press, Oxford, U.K, 1999).

[11] Emergence of superconductivity in heavy-electron materials, Proceedings of the National Academy of Sciences 111:51 18178-18182 (2014), doi:10.1073/pnas.1422100112

[12] Nonlinear lattice dynamics as a basis for enhanced superconductivity in $\mathrm{YBa}_{2} \mathrm{Cu}_{3} \mathrm{O}_{6.5}$. Nature 516 71, DOI: 10.1038 /nature 13875

[13] H. K. Onnes, Communications from the Physical Laboratory of theUniversity of Leiden (1911).

[14] W. Meissner and R. Ochsenfeld, Naturwissenschaften 21, 787 (1933).

[15] F. H. London, Proceedings of the Royal Society of London A 149, 71 (1935).

[16] V. L. Ginzburg, L. D. Landau, and Zh. Eksperim, I Teor. Fiz.20, 1064 (1950).

[17] J. Bardeen, L. N. Cooper, and J. R. Schrieffer, Phys. Rev. 108, 1175 (1957).

[18] W. S. Corak, B. B. Goodman, C. B. Satterthwaite, and A. Wexler, Phys. Rev. 96, 1442 (1954).

[19] W. S. Corak, B. B. Goodman, C. B. Satterthwaite, and A. Wexler, Phys. Rev. 102, 656 (1956).

[20] M. A. Biondi, M. P. Garfunkel, and A. O. McCoubrey, Phys. Rev. 101, 1427 (1956).

[21] R.E. Glover and M. Tinkham, Phys. Rev. 104, 844 (1956).

[22] R.E. Glover and M. Tinkham, Phys. Rev. 108, 243 (1957).

[23] E. Maxwell, Phys. Rev. 78, 477 (1950).
[24] C. A. Reynolds, B. Serin, W. H. Wright, and L. B. Nesbit, Phys. Rev. 78, 487 (1950).

[25] J.G Bednorz, K.A. Mueller (1986). "Possible high $T_{c}$ superconductivity in the Ba-La-Cu-O system" Zeitschrift fur Physik B 64 (2): 189, 193.

[26] D. A. Wollman, D. J. V. Harlingen, W. C. Lee, D. M. Ginsberg, and A. J. Leggett, Phys. Rev. Lett. 71, 2134 (1993).

[27] C. C. Tsuei, J. R. Kirtley, C. C. Chi, L. S. Yu-Jahnes, A. Gupta, T. Shaw, J. Z.Sun, and M. B. Ketchen, Phys. Rev. Lett. 73, 593 (1994).

[28] C. E. Gough, M. S. Colclough, E. M. Forgan, R. G. Jordan, M. Keene, C. M. Muirhead, A. I. M. Rae, N. Thomas, J. S. Abell, and S. Sutton, Nature 326, 855 (1987).

[29] A. Lanzara, P. V. Bogdanov, X. J. Zhou, S. A. Kellar, D. L. Feng,E. D. Lu, T. Yoshida, H. Eisaki, A. Fujimori, K. Kishios, J.-I. Shimoyama, T. Noda, S. Uchida, Z. Hussain, and Z.-X. Shen, Nature 412, 510 (2001).

[30] Ginzburg, V.L. and Landau, L.D. (1950) on the theory of superconductivity. Zhurnal Eksperimentalnoi I Teoreticheskoi Fiziki, 20, 1064-1082. English translation in: L.D. Landau, Collected papers (Oxford: Pergamon Press, 1965).

[31] Josephson, D. (1962) possible new effects in superconductive tunneling. Physics Letter, 1, 251- 253.

[32] Anderson, P.W. and Rowell, J.M. (1963) Probable observation of the Josephson superconducting tunneling effect. Physics Review Letter, 10, 230-232.

[33] Shoji TANAKA "High-Temperature Superconductivity" Japanese Journal of Applied Physics-vol. 45, No. 12, (2006).

[34] Presland, M.R., Tallon, J.L., Buckley, R.G., Liu, R.S., Flower, N.E.: General trends in oxygen stoichiometry effects on Tc in Bi and Tl superconductors. Physica C, Supercond. 176(1-3), 95-105 (1991).

[35] Obertelli, S.D., Cooper, J.R., Tallon, J.L.: Systematics in the thermoelectric power of high-Tc oxides. Phys. Rev. B 46(22), 14928-14931 (1992).

[36] Takagi, H., Batlogg, B., Kao, H.L., Kwo, J., Cava, R.J., Krajewski, J.J., Peck,W.F. Jr.: Systematic evolution of temperature-dependent resistivity in $\mathrm{La}_{2-\mathrm{x}} \mathrm{Sr}_{\mathrm{x}} \mathrm{CuO}_{4}$. Phys. Rev. Lett. 69(20), 2975-2978 (1992)

[37] Marshall, D.S., Dessau, D.S., Loeser, A.G., Park, C.H., Matsuura, A.Y., Eckstein, J.N., Bozovic, I., Fournier, P., Kapitulnik, A., Spicer, W.E., Shen, Z.X.: Unconventional electronicstructure evolution with hole doping in $\mathrm{Bi}_{2} \mathrm{Sr}_{2} \mathrm{CaCu}_{2} \mathrm{O}_{8+\delta}$ : angle-resolved photoemission results. Phys. Rev. Lett. 76(25), 4841-4844 (1996).

[38] Loeser, A.G., Shen, Z.X., Dessau, D.S., Marshall, D.S., Park, C.H., Fournier, P., Kapitulnik, A.: Excitation gap in the normal state of underdoped $\mathrm{Bi}_{2} \mathrm{Sr}_{2} \mathrm{CaCu}_{2} \mathrm{O}_{8+\delta}$. Science 273(5273), 325-329 (1996).

[39] Ding, H., Yokoya, T., Campuzano, J.C., Takahashi, T., Randeria, M., Norman, M.R., Mochiku, T., Kadowaki, K., Giapintzakis, J.: Spectroscopic evidence for a pseudogap in the normal state of underdoped high- $\mathrm{T}_{c}$ superconductors. Nature 382(6586),51-54 (1996). 
[40] Akimitsu, J., Yamazaki, A., Sawa, H., Fujiki, H.: Superconductivity in the $\mathrm{Bi}-\mathrm{Sr}-\mathrm{Cu}-\mathrm{O}$ system. Jpn. J. Appl. Phys. 26(Part 2, 12), L2080-L2081 (1987).

[41] Maeda, H., Tanaka, Y., Fukutomi, M., Asano, T.: A new highTc oxide superconductor without a rare earth element. Jpn. J. Appl. Phys. 27(Part 2, 2), L209-L210 (1988).

[42] Pickett, W.E.: Electronic structure of the high-temperature oxide superconductors. Rev. Mod.Phys. 61(2), 433-512 (1989).

[43] A. Sedky, A. A. Almulhem, and S. S. Ibrahim, Smart Mater. Struct. 15, N99 (2006).

[44] M.K. Wu, J.R. Ashburn, C.J. Torng, P.H. Hor, R.L. Meng, L. Gao, Z.J. Huang, Y.Q. Wang, and C.W. Chu (1987). Physical Review Letters 58 (9): 908-910.

[45] C. Michel et al., Z. Phys. B68, 421 (1987).

[46] H. Maeda et al., Jpn. J. Appl. Phys. 27, L209 (1988).

[47] J.M. Jarascan et al., Phys. Rev. B37, 9382 (1988).

[48] S.A. Sunshine et al., Phys. Rev. B38, 893 (1988).

[49] J.M. Tarascan et al., Phys. Rev. B38, 8885 (1988).

[50] C. Politis, Appl. Phys. A 45, 261 (1988).

[51] Z. Z. Sheng and A. M. Hermann, Nature 332, 55 (1988).

[52] Z. Z. Sheng, A. M. Hermann, A. E. Ali, C. Almasan, J. Estrada, T. Datta, and R. J. Matson, Phys. Rev. Lett. 60, 937 (1988).

[53] Z. Z. Sheng and A. M. Hermann, Nature 332, 138 (1988).

[54] G. Malandrino, D. S. Richeson, T. J. Marks, Donald C. De Groot, J. L. Schindler, and C. R. Kannewurf, Appl. Phys. Lett. 58, 182 (1991).

[55] M. L. Chu, H. L. Chang, C. Wang, J. Y. Juang, T. M. Uen, and Y. S. Gou, Appl. Phys. Lett. 59, 1123 (1991).
[56] W. L. Oslon, M. M. Eddy, T. W. James, R. B. Hammond, G. Gruner, and L. Drabeck, Appl. Phys. Lett. 55, 188 (1989).

[57] Sheng, Z. Z.; Hermann, A. M.; El Ali, A; Almasan, C; Estrada, J; Datta, T; Matson, R. J. (1988). "Superconductivity at $90 \mathrm{~K}$ in the Tl-Ba-Cu-O system" Physical Review Letters 60(10): 937-940.

[58] Sheng, Z. Z.; Hermann, A. M. (1988). "Superconductivity in the rare-earth-free $\mathrm{Tl}-\mathrm{Ba}-\mathrm{Cu}-\mathrm{O}$ system above liquid-nitrogen temperature". Nature- 332 (6159) 55.

[59] S.N. Putilin et al., Mater. Res. Bull. 26, 1299 (1991).

[60] S.S.P. Parkin et al., Phys. Rev. B38, 6531 (1988).

[61] S.N. Putilin et al., Nature 362, 226 (1993).

[62] S.N. Putilin et al., Physica C212, 266 (1993).

[63] A. Schlling et al., Nature 363, 56 (1993).

[64] D. J. Singh and M.-H. Du, Phys. Rev. Lett. 100, 237003 (2008).

[65] M. G. Zhao and S. Y. Wang, Cond-Mat/0111268v1 (2001).

[66] J.R. Hull, Rep. Prog. Phys. 66, 1865 (2003)

[67] J.N.A. Matthews, Physics Today 61, 30 (2008)

[68] A.P. Malozemoff, S. Fleshler, M. Rupich, C. Thieme, X. Li, W. Zhang, A. Otto, J. Maguire, D. Folts, J. Yuan, H.-P. Kraemer, W. Schmidt, M. Wohlfart, H.-W. Neumueller, Supercond. Sci. Technol. 21, 034005 (2008).

[69] V. Selvamanickam, Y. Chen, X. Xiong, Y. Xie, X. Zhang, Y. Qiao, J. Reeves, A. Rar, R. Schmidt, K. Lenseth, Physica C 463-465, 482 (2007).

[70] T. Matsushita, M. Kiuchi, K. Kimura, S. Takayama, Y. Yamada, Y. Shiohara, J. Fujikami, K. Hayashi, K. Sato, Physica C 463-465, 686 (2007). 